\title{
Topographic and thermal mapping of volcanic terrain using AVTIS ground-based 94-GHz dual-mode radar/radiometric imager
}

Article

Published Version

Macfarlane, D. G., Odbert, H. M., Robertson, D. A., James, M. R., Pinkerton, H. and Wadge, G. (2013) Topographic and thermal mapping of volcanic terrain using AVTIS ground-based 94-GHz dual-mode radar/radiometric imager. IEEE

Transactions on Geoscience and Remote Sensing, 51 (1). pp. 455-472. ISSN 0196-2892 doi:

https://doi.org/10.1109/TGRS.2012.2202667 Available at https://centaur.reading.ac.uk/30603/

It is advisable to refer to the publisher's version if you intend to cite from the work. See Guidance on citing.

To link to this article DOI: http://dx.doi.org/10.1109/TGRS.2012.2202667

Publisher: IEEE Geoscience and Remote Sensing Society

All outputs in CentAUR are protected by Intellectual Property Rights law, including copyright law. Copyright and IPR is retained by the creators or other copyright holders. Terms and conditions for use of this material are defined in the End User Agreement. 


\section{www.reading.ac.uk/centaur}

\section{CentAUR}

Central Archive at the University of Reading

Reading's research outputs online 


\title{
Topographic and thermal mapping of volcanic terrain using the AVTIS ground based $94 \mathrm{GHz}$ dual-mode radar/radiometric imager
}

\author{
David G. Macfarlane, Member, IEEE, Henry M. Odbert, Duncan A. Robertson, Member, \\ IEEE, Mike R. James, Harry Pinkerton, and Geoff Wadge
}

\begin{abstract}
The AVTIS remote sensing instrument is a custom built millimeter wave sensor that has been developed as a practical field tool for remote sensing of volcanic terrain at active lava domes. The portable instrument combines active and passive millimeter wave measurements to record topographic and thermal data in almost all weather conditions from ground-based survey points. We describe how the instrument is deployed in the field, the quality of the primary ranging and radiometric measurements and the post-processing techniques used to derive the geophysical products of the target terrain, surface temperature and reflectivity. By comparison of changing topography we estimate volume change and lava extrusion rate. Validation of the millimeter wave radiometry is also presented by quantitative comparison with coincident infrared thermal imagery.
\end{abstract}

Index Terms - Digital elevation models, Millimeter wave propagation, Millimeter wave radar, Millimeter wave imaging, Radiometry, Terrain Mapping, Volcanoes

\section{INTRODUCTION}

$\mathrm{D}$ IRECT observations of active volcanoes are often severely restricted by environmental conditions, particularly cloud cover, and visible observations of volcanic activity at the summit of a volcano may be impossible or very sparse for days to weeks. This can be a major impediment to detecting potential hazards derived from unseen activity. The

Manuscript received August 26, 2011; revised January 19 2012; accepted April 18 2012. This work was supported the UK Natural Environment Research Council (NERC) under several successive grants since 2002: NER/A/S/2001/01001, NE/D00859X/1, NE/E001734/1 and NE/E015352/1.

D. G. Macfarlane is with the School of Physics and Astronomy, University of St Andrews, St Andrews, Fife, KY16 9SS, UK (phone: (44)1334-461608; fax: (44)-1334-467307; e-mail: dgm5@st-and.ac.uk ).

H. M. Odbert was with the Environmental Systems Science Centre (ESSC), Reading University, UK. He is now with the Montserrat Volcano Observatory, Montserrat, W.I. (e-mail: henryodbert@googlemail.com).

D. A. Robertson is with the School of Physics and Astronomy in St Andrews (e-mail: dar@st-and.ac.uk).

M. R. James and H. Pinkerton are with the Lancaster Environment Centre, Lancaster University, Lancaster, U.K. (e-mail: m.james@lancs.ac.uk; h.pinkerton@lancs.ac.uk).

G Wadge is with the ESSC, Reading University, U.K. (e-mail: g.wadge@reading.ac.uk) need to overcome this problem at the Soufrière Hills Volcano on the island of Montserrat was a prime motivation for the creation of the AVTIS (All-weather Volcano Topography Imaging Sensor) instrument.

The Soufrière Hills Volcano has been building large (up to $400 \times 1000 \mathrm{~m}$ ) lava domes over varying intervals since 1995 . During periods of high activity lava extrusion can alter the dome topography at a rate of meters per day. The dome grows and collapses repeatedly with all but the smallest rockfall collapse events producing pyroclastic flows. In the first few years of eruption survey techniques such as photogrammetry, total station measurements, and laser ranging binoculars were used to estimate the changing topography of the growing lava dome and from these measurements, volumetric change and flux were calculated [1]. More recently, techniques such as ground-based lidar [2] and hand-held infra-red cameras [3] have begun to be used for active volcano survey and monitoring but these are often limited by severe to total attenuation due to cloud.

The average repeat frequency of surveys achieved on Montserrat over the years using these techniques has been about 20 to 30 days however the timing, direction and magnitude of dome collapse pyroclastic flows remain unpredictable. The hazard posed by the volcano increases under certain conditions, for example when lava intrudes within the dome rather than reaches the surface [4], and knowledge of changing terrain shape is potentially of great value in forecasting future hazardous behavior. This should be detectable by topographic surveys but, given their infrequency, is not generally feasible or reliably done by traditional techniques.

Remote sensing techniques that can record topography during periods of poor visibility include satellite and airborne interferometric synthetic aperture radar (InSAR) [5] which can produce digital elevation maps (DEMs) with a few meters height resolution, extensive coverage and sensitivity to small changes in topography. However, they require coherence between repeat passes (typically days/months) which is easily lost on the rapidly evolving topography of active lava domes rendering the technique useless for monitoring the regions of greatest interest [6].

High temporal frequency observation of volcanoes can more readily be achieved with ground based microwave radar 
systems and have included utilizing permanent ground based high power pulsed weather radar in Iceland to probe eruption plumes at long range $(\sim 300 \mathrm{~km})$ [7], portable low power continuous wave radar to measure eruption projectile and rockfall velocities at Merapi over ranges of 4 to $6 \mathrm{~km} \mathrm{[8]} \mathrm{and}$ ground based InSAR to measure surface deformation at Stromboli at ranges of up to $1.2 \mathrm{~km}$ [9]. Of these, only ground based InSAR provides topographic information. It does not produce a DEM but reveals small surface displacements $(\mathrm{mm})$ by comparing successive phase maps and is only suitable for a fairly narrow range of surface deformation rates, not for measuring the emplacement of new lava. Ground based SAR can be used to record DEMs but this either requires a separated pair of antennas or a repeat pass observation with non-zero baseline [10].

The primary aim of the AVTIS instrument is to enable round-the-clock monitoring of lava dome bulk growth, i.e. detect topographic changes on the order of meters per day. The sensor is designed for operation as a ground-based portable remote sensing instrument allowing survey from multiple viewpoints to be carried out in the field at short notice. AVTIS is a dual mode passive and active millimeter wave (MMW) imaging sensor. MMW instruments are physically smaller than their microwave counterparts of the same spatial resolution, and whilst more susceptible to atmospheric attenuation than microwave sensors they can penetrate atmospheric conditions which are opaque to the visible and infrared. In passive mode AVTIS acts as a radiometric receiver mechanically rastering a single antenna pencil beam across the field of view to build up a thermal image of the volcano through obscuring gas and cloud. In active mode AVTIS rasters the beam across the scene using continuous wave radar to measure the range to the surface for each direction thus building a DEM. Power reflected from the surface is also recorded as a by-product of the radar ranging measurement.

AVTIS has been used to make measurements on four campaigns at Soufrière Hills Volcano and once at Arenal Volcano in Costa Rica and the scientific results derived from those measurements are reported elsewhere [11] - [15]. In this paper we start by describing the basic properties of the instrument and how it is deployed in the field (Section II) and the process of generating a georeferenced DEM with AVTIS data (Section III). Following this we examine long range radar performance (Section IV), and characterization (Section V) leading to terrain radar cross section analysis (Section VI) and examples of DEMs captured and differenced with AVTIS (section VII). We finish with a validation of MMW radiometric data compared to coincident infrared imagery (Section VIII) before drawing conclusions in Section IX.

\section{SYSTEM DESCRIPTION}

\section{A. General Description}

The AVTIS system, shown in Fig. 1, is a gimbal-mounted instrument fitted with a single $0.3 \mathrm{~m}$ diameter Cassegrain antenna, which can be fitted to a conventional surveyor's

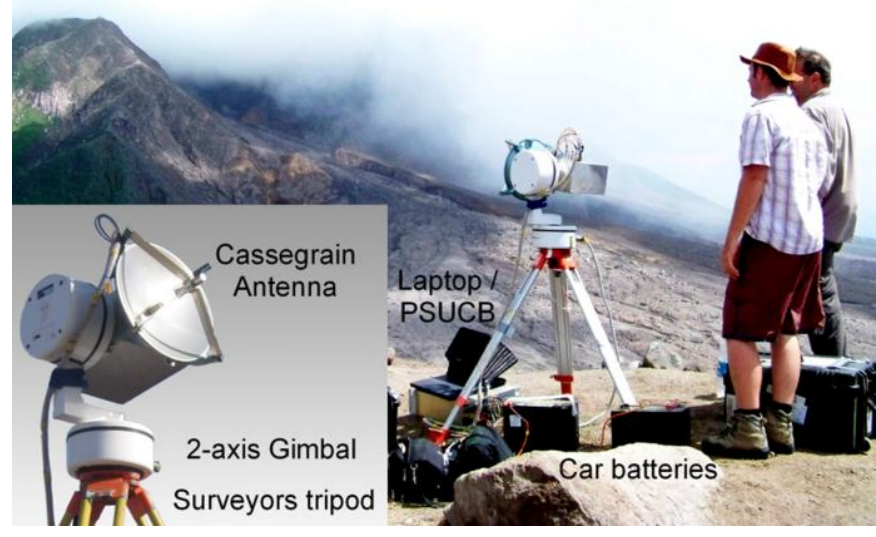

Fig. 1. The AVTIS $94 \mathrm{GHz}$ radar/radiometric instrument set up for use in the field on Montserrat, W.I. The lava dome being scanned is obscured by the volcanic gas plume and lies behind the foreground ridge.

tripod or to a fixed monument. The antenna and gimbal are connected to a power supply unit and control box (PSUCB) and to a laptop computer that interfaces with the whole system to measure and record data. The instrument head and control gimbal are each powered by a standard $12 \mathrm{~V}$ car battery with the laptop computer running from its own internal batteries. The whole system can be readily transported by car or helicopter to a suitable surveying site and is typically set up and operated by two people.

Data acquisition can be performed in either passive radiometric mode or active radar mode, but the two measurements cannot be performed simultaneously. Each measurement can be taken for a single line of sight (LOS), with a scene of interest being covered by repeating measurements over an angular raster grid (a 'scan').

Radar measurement points are inherently of range to terrain as a function of pointing angle and are converted into georeferenced Cartesian coordinates and heights for each DEM. Repeat topographic measurements then allow volume change to be measured by differencing the height of successive DEMs. Changes in topography are most sensitive in the range direction due to the high range resolution of the radar, which remains constant for all ranges, whilst changes in azimuth and elevation (which correspond to height changes in the DEM) are resolved by the antenna angular beamwidth, which is a function of range.

Data acquisition and storage is semi-automated with sequential scans being stacked to enable averaging over a single field setup. Although basic data visualization is available to the user in real time, data calibration, terrain reconstruction and referencing to a local geographic coordinate system are carried out in offline post-processing.

Table 1 gives a summary of system parameters and performance. The two-way $3 \mathrm{~dB}$ radar beamwidth is $0.52^{\circ}$ which at normal incident translates to spot diameter of 9.1 $\mathrm{m} / \mathrm{km}$, scaling linearly with distance. If the terrain is inclined, the projected area of the beam footprint will be approximately elliptical (eg $12.9 \times 9.1 \mathrm{~m} / \mathrm{km}$ at $45^{\circ}$ incidence). A typical field of view is $20^{\circ} \times 5^{\circ}$ (which represents an area of $349 \times 87$ $\mathrm{m} / \mathrm{km}$ at normal incidence) sampled at $0.1^{\circ}$ intervals 


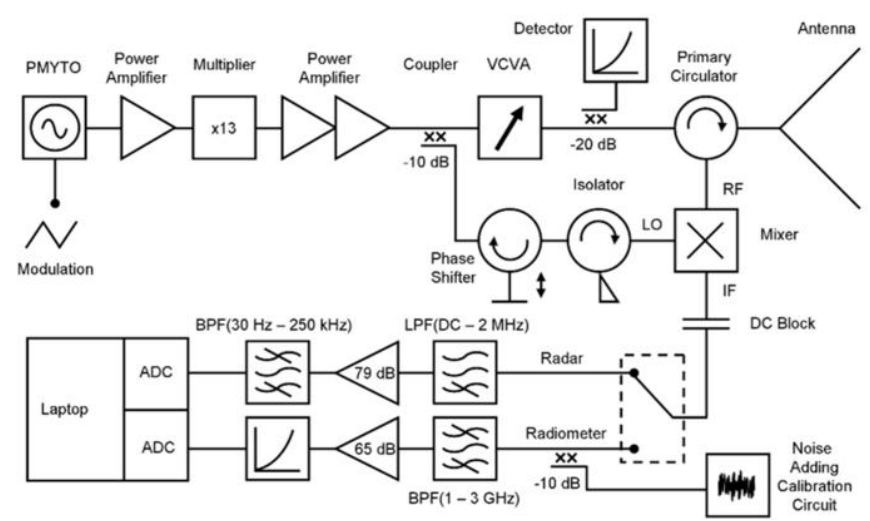

Fig. 2. AVTIS sensor head architecture. The instrument is a heterodyne receiver with radar and radiometer receivers sharing the same mixer. Active and passive measurements cannot be performed simultaneously and are selected by switching the transmit power and receiver chains appropriately.

containing 10,000 lines of sight.

\section{B. Technical Description of Sensor Head}

The AVTIS sensor head combines a heterodyne radiometer with a homodyne, frequency modulated continuous wave (FMCW) radar. The sensor head layout is shown in Fig. 2. AVTIS uses a frequency multiplied $7.23 \mathrm{GHz}$ oscillator to provide a single $94 \mathrm{GHz}$ source as both the radar transmitter and the local oscillator (LO) for the radiometer. Active and passive modes are selected by attenuating the transmit path by $55 \mathrm{~dB}$ using a voltage controlled variable attenuator (VCVA) when in radiometric mode, switching between the appropriate intermediate frequency (IF) channels and choosing either triangular source modulation (for radar) or no modulation (for radiometer). A more detailed description of the sensor head and performance can be found elsewhere [15].

\section{Field Setup}

AVTIS is transported to the field and the head is assembled and powered in about five minutes. Thermal stabilization of the head takes a further forty five minutes. This time is used to accurately determine the instrument pointing. The tripod is leveled and the gimbal attached. The gimbal self-calibrates by rotating in sequence to the extreme positions of azimuth and elevation. The head is then attached and the whole instrument manually rotated on the tripod to point at a reference corner cube (CC) reflector deployed in the field at a position measured by differential GPS (dGPS) at ranges of up to $\sim 2 \mathrm{~km}$ from the radar. Once the $\mathrm{CC}$ has been bore sighted in the radar frame of reference, the gimbal is locked in position. Typically, two or three CCs are used with different azimuths and ranges. For repeat surveys, this allows matching of the orientation of the gimbal as closely as possible to previous occupations.

Once AVTIS has been installed at the surveying location, it can be operated in a semi-autonomous fashion, repeating raster scans with pre-loaded parameters until stopped. In between each raster, repeated measurement of the CCs is required to calibrate thermal drift in the radar range accuracy. Measurements of the atmospheric brightness temperature versus elevation are also required alongside each radiometric raster. The longest continuous operation of AVTIS to date has
TABLE I

SUMMARY OF SYSTEM PERFORMANCE

\begin{tabular}{|c|c|}
\hline System Parameter & Value \\
\hline \multicolumn{2}{|c|}{ General } \\
\hline Centre Frequency & $94 \mathrm{GHz}$ \\
\hline Antenna Diameter & $300 \mathrm{~mm}$ \\
\hline Antenna Gain & $46.2 \mathrm{~dB}$ \\
\hline Sidelobe Level (one-way) & $-20 \mathrm{~dB}$ \\
\hline Instrument Ready Time & 5 min plus 45 min warm up \\
\hline Imaging Time $\left(20^{\circ} \times 5^{\circ}, 0.1^{\circ}\right.$ inc. $)$ & $50 \mathrm{~min}$ \\
\hline Weight & $40 \mathrm{~kg}$ excluding car batteries \\
\hline Portability & 2 people in helicopter/jeep \\
\hline Operational Duration & 8 hours per battery charge \\
\hline \multicolumn{2}{|c|}{ Radar Mode } \\
\hline Radar IF Band & $30 \mathrm{~Hz}-250 \mathrm{kHz}$ \\
\hline FM Chirp Time & $32 \mathrm{~ms}$ \\
\hline Max. Operational Range & $\sim 6500 \mathrm{~m}$ \\
\hline Polarization & VV \\
\hline Transmit Power & $20.5 \mathrm{dBm}$ \\
\hline Receiver Noise Figure & $10 \mathrm{~dB}$ \\
\hline SSB Phase Noise at $10 \mathrm{kHz}$ & $-73 \mathrm{dBc} / \mathrm{Hz}$ \\
\hline RF chirp bandwidth & $176.8 \mathrm{MHz}$ \\
\hline FFT Range resolution & $0.85 \mathrm{~m}$ \\
\hline Two-way $3 \mathrm{~dB}$ Beamwidth & $0.52^{\circ}$ \\
\hline Nominal Radar Pixel Size & $0.1^{\mathrm{o}} \times 0.1^{\mathrm{o}}$ \\
\hline \multicolumn{2}{|c|}{ Radiometer Mode } \\
\hline Radiometer IF Band & $1-3 \mathrm{GHz}$ \\
\hline Receiver Noise Temperature & $6000 \mathrm{~K}$ \\
\hline Thermal Sensitivity & $<5 \mathrm{~K}$ \\
\hline Integration Time & $32 \mathrm{~ms}$ \\
\hline One-way 3 dB Beamwidth & $0.74^{\circ}$ \\
\hline Nominal Radiometer Pixel Size & $0.2^{\circ} \times 0.2^{\mathrm{o}}$ \\
\hline
\end{tabular}

been about eight hours, and was eventually limited by the PSUCB battery. Where possible, a lightweight tent is used to protect AVTIS from overheating in the sun and from being buffeted by wind.

\section{Generating A Digital Elevation Model}

\section{A. Overview}

The process for generating a georeferenced DEM has been developed over the last nine years from a time consuming manual analysis of the raw data that would take many months to produce complete and accurate results, to a semiautonomous process which can provide a DEM a few minutes after data acquisition. Each raw AVTIS radar dataset consists of a rastered set of range spectra with one or more FMCW chirp (or frequency sweep) measurements taken for each LOS. This volumetric set of range versus reflected power spectra is then corrected for frequency dependent gain in the receiver IF chain. In addition, since the incident radar beam on the target scene topography can extend over many tens of meters (especially for oblique surfaces) and can be dominated by a strongly reflecting sub-beamwidth facet (e.g. a large perpendicular boulder) the spectra are low-pass filtered to provide a measurement of average radar reflectivity for the bulk topography. For longer ranges $(>4 \mathrm{~km})$ averaging of successive scans can be required to enhance signal-to-noise. Topography is then extracted from this processed volume dataset by locating a single range at the point of maximum reflectivity for each LOS. The resulting set of data points is amplitude corrected for fall-off with range and thresholded in 
TABLE II

DATA PROCESSING AND STORAGE HIERARCHY FOR EACH SETUP OF AVTIS

\section{OCCUPATION}

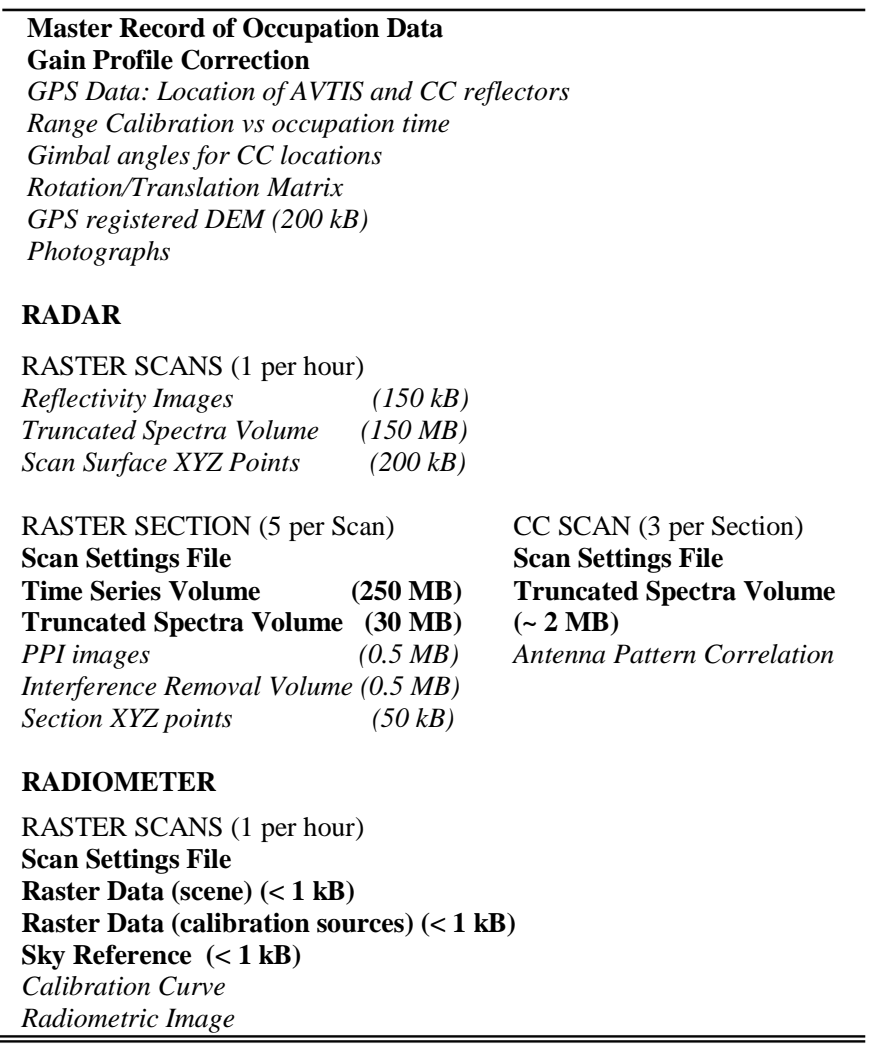

N.B.: objects in bold denote field data, objects in italics denote postprocessing data products

amplitude to discard LOS with no discernible return; these occur at the edges of ridges and clear sky.

Registration of the data to a local geographic coordinate system is achieved by locating several different CC reflectors in the radar frame of reference. Combining these vectors with the dGPS-determined positions of the instrument and CCs then provides the translation and rotation transforms to georeference the radar data. Iterative minimization of DEM differences in areas of topography known to be static is also used as a final refinement on the absolute orientation of the radar-derived topography within a georeferenced grid system.

Radiometric measurements use a two-stage calibration process with short term gain fluctuations in the receiver calibrated against an internal noise source for each LOS in a raster. Absolute temperature calibration is achieved by reference to the thermal temperature gradient of the atmosphere. Co-locating the thermal data with the radar data is then trivial since the measurements share the same antenna.

\section{B. Detailed DEM Extraction Process}

There now follows a more detailed description of the steps that are taken to create a georeferenced DEM using AVTIS. Data capture in the field is described in the first two steps, with the remaining steps carried out in post-processing.
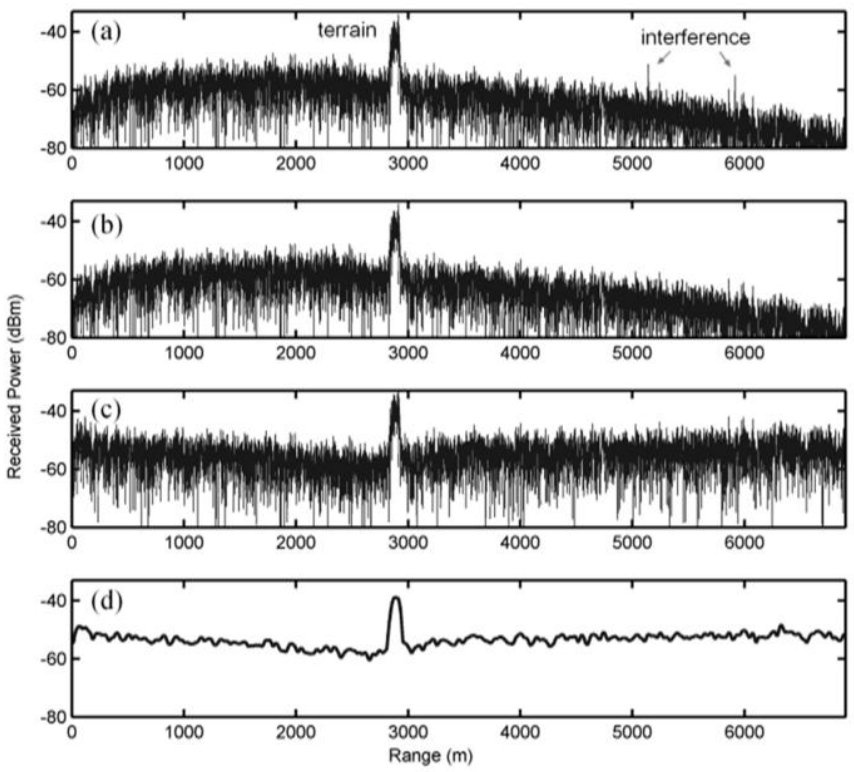

Fig. 3. FMCW spectrum processing sequence: (a) raw data for a single line of sight, (b) interference removed, (c) corrected for IF Gain, and (d) low pass filtered.

1) Data capture: FMCW chirp capture for each LOS in a rastered azimuth - elevation scan

FMCW radar uses a linear frequency chirp in transmit which is mixed against the delayed copy of itself returning from the scene. The resulting spectrum gives reflected power where range, $R$, is proportional to the beat frequency, $f_{I F}$ :

$$
R=\frac{f_{I F} c T_{S}}{2 B},
$$

where $T_{S}$ denotes the FMCW chirp time and $B$ is the chirp bandwidth. Note that this means that any frequency dependence in the radar receiver can equivalently be thought of as range dependence. AVTIS uses a $32 \mathrm{~ms}$ chirp with a 175 $\mathrm{MHz}$ bandwidth centered at $94 \mathrm{GHz}$, giving a range resolution of $c / 2 B=0.85 \mathrm{~m}$.

The frequency chirp for each FMCW measurement in AVTIS is set by a triangular modulation voltage applied to the oscillator and is supplied by an analogue waveform generator which also produces a simultaneous digital trigger signal for timing data acquisition. This repetitive modulation is free running and is independent of the gimbal pointing. For each LOS a 12-bit, 16384-point time series data sample is recorded, Hann weighted and converted to a frequency spectrum using a fast Fourier transform (FFT). For a fixed LOS any number of successive chirps can be averaged.

For a raster scan across a scene of interest the gimbal moves the antenna to successive azimuth and elevation LOS positions, pausing at each LOS to sample on the next available trigger. A typical volcanic scene takes up to an hour to acquire, which is sufficiently fast for day-to-day surveying. However this process is not particularly efficient, taking around half a second per LOS, and could be improved by synchronizing the radar modulation to the gimbal movement. 

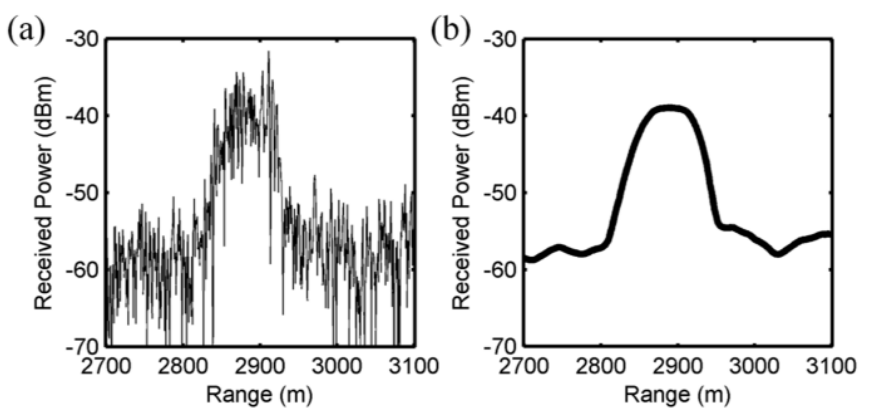

Fig. 4. Example of truncated range spectrum: (a) spectra are truncated to save only the ranges of interest to reduce data storage and processing requirements, (b) the filtered spectra gives a better estimate of the range to terrain at the boresight of the incident beam. Unfiltered data can give a range to maximum return due to dominant scatterers that differs by tens of meters from the boresight range.

\section{2) Data capture: Stacking of successive raster scans within each scene}

Data are organized and stored hierarchically with each individual setup of the instrument saved as a separate occupation with a master record of the data capture stored at the root level of the data hierarchy (Table II).

Here we describe the data structure for a typical raster scene size of a $20^{\circ} \times 5^{\circ}$ scan (azimuth $\times$ elevation) sampled at $0.1^{\circ}$ increments, taking 50 minutes to acquire. This raster corresponds to 10000 LOS which results in a total raw data size of $\sim 1.3 \mathrm{~GB}$. This dataset size is too large for the controlling computer to process and would also lead to data storage problems. Thus, each raster is broken down into manageable sections with a maximum size of $\sim 250 \mathrm{MB}$.

In practice, all these data do not always need to be stored. The power spectra volume after Fourier transformation is half the size ( 125 MB) and can be truncated to record only the ranges of interest with the scene. Typically this is about a quarter of the entire spectrum, $\sim 30 \mathrm{MB}$. However, the data storage structure is based on the requirement to save the full time series dataset since this allows development of analytical tools for AVTIS data beyond the initial requirement of surface retrieval (e.g. Doppler analysis). Note that the data storage requirements for the radiometric data are negligible since there are only three values recorded for each LOS (azimuth, elevation, temperature).

Thus the raster is broken down into 5 sections of $20^{\circ} \times 1^{\circ}$. Data are saved in binary format with each section labeled as being part of a larger scan for post-processing purposes. The interval between each section is also used for reference CC scans (see Fig. 6) with up to three separate CC scans at each section interval. The scan settings for each scene and CC scan are saved with the data and the raster repeated in an automated sequence.

\section{3) Post-Processing: Basic data spectra}

Post-processing is carried out using a custom written MATLAB script operating on data at the root level of each occupation. The application of each of the following steps is recorded and saved at the appropriate hierarchical level such that the processing routine does not repeat a previously calculated process (e.g. interference removal) unless

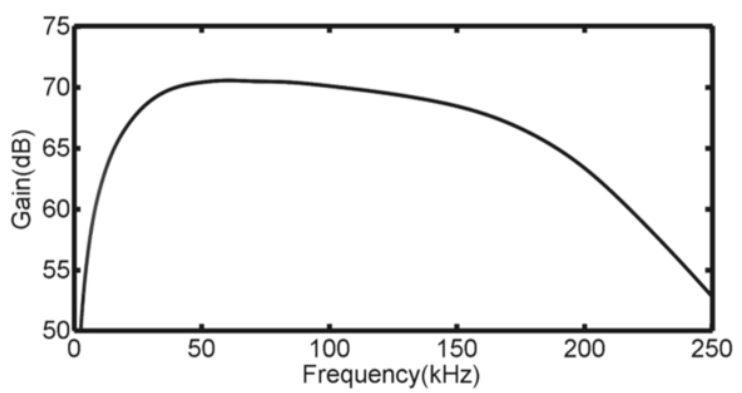

Fig. 5. AVTIS radar receiver gain profile. The non-uniform gain is accounted for when assessing radar returns from different ranges. The gradual anti-aliasing filter is sufficient since the signal strength of real returns from ranges beyond Nyquist cutoff $(250 \mathrm{kHz})$ is insufficient to produce any aliasing effects.

specifically requested to do so. This avoids inefficient repetition of time consuming processing tasks.

Processing is carried out on each raster section in sequence. For each LOS in a raster section, the first step is to produce the range spectrum, Fig. 3 (a), which shows a full range spectrum for a LOS that intercepts volcanic terrain. To reduce data storage requirements this spectrum is truncated to a region a few hundred meters either side of the terrain, such as in Fig. 4 (a). Truncation limits are set manually, based on user judgment. In Fig. 3, the entire spectrum is shown to illustrate the effects of post-processing corrections.

\section{4) Post-processing: Removal of interference}

Electronic interference is removed from the spectra at this stage, Fig. 3 (b). Interference removal is essential to avoid errors in DEM surface construction especially at longer ranges where the magnitude of interference can be comparable to the target returns. We use an automated algorithm to remove discrete interference lines based on analysis of long range field data (see section IV). This was a time consuming process to develop and marginal data (those with low SNR recorded during heavy cloud or light rain) still require some manual inspection, however non-marginal cases are dealt with robustly. Any corrections to remove interference from the spectra volume are saved as a separate dataset which are automatically applied to the spectra on any future analysis, thus preserving the raw data.

\section{5) Post-processing: Correction of spectra for gain profile}

The next step is to correct each LOS range spectrum for the frequency dependent gain profile of the AVTIS radar receiver. The laboratory characterization of the receiver gain is shown in Fig. 5. The AVTIS receiver gain profile is not particularly flat across the radar IF primarily due to the effects of a DC blocking capacitor on the mixer output and a gradual $250 \mathrm{kHz}$ anti-aliasing filter. Compensating for this profile both flattens the noise floor and boosts long range returns in the spectrum data, Fig. 3 (c).

\section{6) Post-processing: Filtering of spectra and surface extraction}

The range to the target terrain now corresponds to the frequency bin containing the highest returned power. Ideally the target would be a sharply-defined peak in the spectrum 


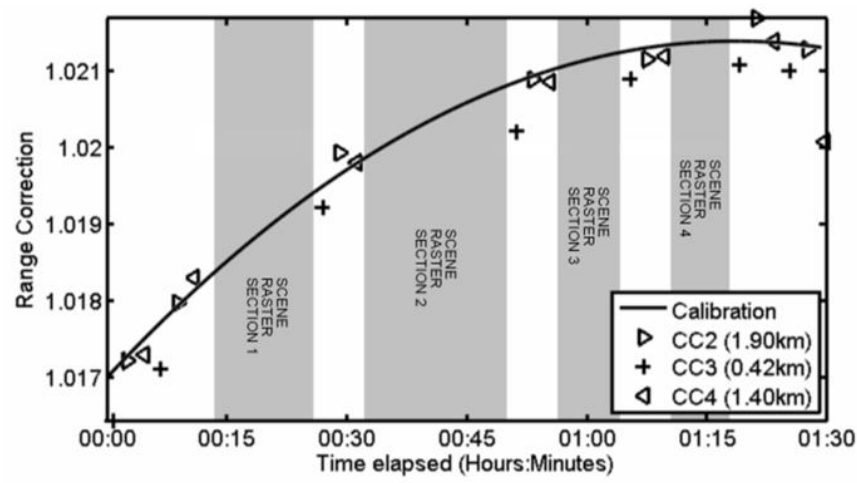

Fig. 6. Thermal drift in radar response. The proportional change in apparent range to fixed $\mathrm{CC}$ reference reflectors is shown as a function of time from switch on of the instrument. The calibration fit used to correct the scene data is based on $\mathrm{CC} 2$ and $\mathrm{CC} 4$ data since they lie at a far greater range than $\mathrm{CC} 3$. The grey areas show the times during which terrain data was captured.

against the background noise. In reality, the radar beam is typically incident across uneven and inclined terrain resulting in the power being reflected from multiple scatterers spread over many tens of meters in range. Coherent speckle causes the range to maxima in the raw spectra to fluctuate by tens of meters from the boresight range value as returns from scatterers of similar amplitude interact within the beam footprint [16]. Recording multiple FMCW sweeps for each LOS can reduce noise and lessen fluctuations but the overall time for a single raster lengthens to many hours and this is impractical to implement in the field. We instead apply a twoway rolling filter to the spectrum to reduce high frequency fluctuations and yield the range to terrain at the boresight of the antenna beam, Fig. 4 (b). Bulk movement of the surface contained within the beam footprint is resolvable at the FFT resolution of $0.85 \mathrm{~m}$. The optimum filter length is likely to be related to the incident beam footprint size which is dependent on range to scene and inclination of terrain (see section VI). However, empirical analysis of field data showed that a filter width of 36 spectral bins yields optimum range stability between successive measurements of the same LOS.

The filtered spectra volume is then converted into a spherical coordinate point dataset, recording the range and reflected power at the maximum of each LOS in the scene raster.

\section{7) Post-processing: Correction for range drift over time}

During AVTIS operation, the apparent range to a target changes slowly over time in line with thermal changes in the active components within the instrument head. As the temperature changes, the chirp time alters due to thermal drift in the waveform generator circuit. From (1) this produces an associated drift in the measured range.

The range measurement takes around 45 minutes to stabilize from turn on, after which the residual variation is $\sim 0.2 \%$ over an hour timescale, Fig. 6 . This corresponds to a 10 $\mathrm{m}$ shift in apparent range for terrain at $5 \mathrm{~km}$. To calibrate for this drift we regularly record the radar derived range to reference $\mathrm{CC}$ reflectors at fixed positions of known range from the radar as measured by dGPS and use this to rescale the radar range measurement. The $\mathrm{CC}$ reflectors are stainless steel trihedrals with a radar cross section (RCS) of $+20 \mathrm{dBsm}$ and can be detected above clutter at ranges of up to $\sim 2 \mathrm{~km}$. The $0.2 \%$ drift implies that any $\mathrm{CC}$ reference reflector at a range of greater than $425 \mathrm{~m}$ can drift by more than the radar FFT bin resolution of $0.85 \mathrm{~m}$. The $\mathrm{CC}$ range is determined to sub-bin resolution by fitting a polynomial to the peak of the CC return. To improve the quality of the fit, we typically use an average of 221 LOS produced by a $1^{\circ} \times 1^{\circ}$ raster of the CC taken for pointing calibration (discussed below in step 10).

The AVTIS processing code has been developed to automatically load corner-cube scan data and record the range to the reflector for each scan as the average of the fitted range for these 221 LOS. The ratio between the radar derived range and the dGPS range is recorded as a function of time over the course of each occupation and is saved at the root level of the data hierarchy. A calibration curve is fitted to these data and used to correct the range associated with each point in the 3D scene data depending on the time of acquisition (calculated from start-time signatures contained in the metadata file of each scene raster). Fitting the calibration curve has not yet been automated since the characteristic shape of range drift with time varies from occupation to occupation due to variability in environmental conditions subtly altering the refractive index of the atmosphere in the radar LOS. Further work is required to quantify the level of impact of these variations on the AVTIS radar range measurement.

\section{8) Post-processing: Conversion to Cartesian coordinates}

At this point the data is converted to a cartesian frame of reference with the radar at the origin using a standard polar transformation.

\section{9) Post-processing: Thresholding of low return LOS}

Due to the ground based nature of AVTIS surveying, some proportion of a rastered scene usually incorporates 'sky' returns where the LOS does not intersect any topography. In such cases the LOS maxima yield spurious low-power points from the spectrum noise floors that do not correspond to the target terrain, Fig. 7 (a). To remove these points we first convert the reflected power associated with each point to normalized RCS (RCS per unit area). This conversion mainly accounts for fading in returned power with range and is nontrivial. A more detailed discussion of this conversion process is given in section VI.

Histograms of RCS values then show a clear distinction between terrain and 'sky' data points, Fig. 7 (b). By choosing a fixed RCS threshold for the entire data, low value points are removed and the terrain can be plotted, Fig. 7 (c). We attempted to standardize this process based on Gaussian fits to the RCS distributions but this did not prove to be robust since occupations with poor atmospheric conditions (heavy cloud and light rain especially) lessen the distinction between the 'sky' and terrain distributions. In practice, thresholds have been chosen by user inspection of RCS histograms on a case by case basis, with the chosen threshold held fixed for each occupation. 

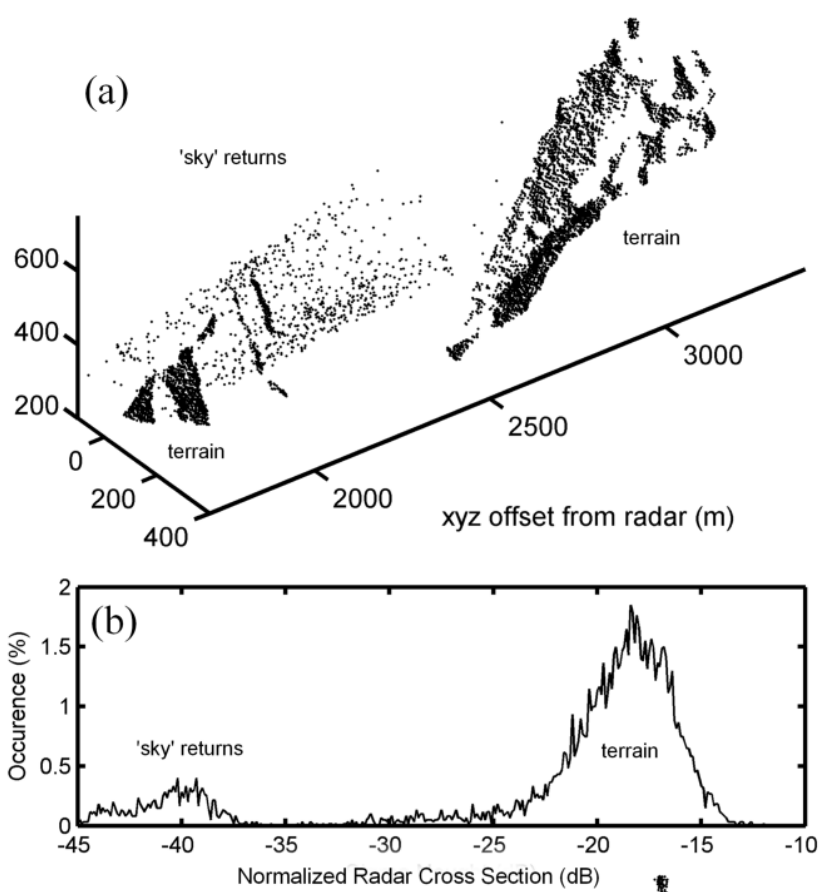

(c)

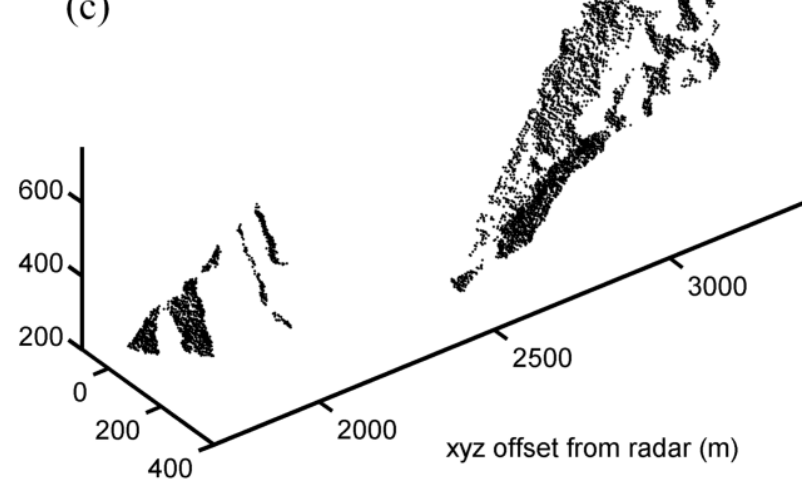

Fig. 7. 3D topographic point cloud in the radar frame of reference. (a) Topography with spurious 'sky' returns corresponding to LOS that do not intercept terrain, (b) histogram of normalized RCS (RCS per unit area) for the target terrain with points less than $-32 \mathrm{~dB}$ removed from the data, and (c) topographic point cloud after RCS thresholding.

\section{0) Post-processing: Georeferencing radar data}

The next stage is to register and transform the radar data (in spherical coordinates) to the local geographic coordinate system, through the use of reference CCs at fixed locations. This requires knowledge of the absolute position and orientation of the AVTIS gimbal. The positions of the AVTIS radar and multiple $\mathrm{CC}$ references are therefore recorded with dGPS during each occupation. To reduce errors in constraining angular positions, CCs are ideally deployed at positions that offer the widest separation possible in azimuth and elevation and as far as possible from the radar, up to a range of $\sim 2 \mathrm{~km}$.

Within the radar coordinate system, the angular position of each $\mathrm{CC}$ is obtained by correlating the known two-way antenna pattern of the instrument, Fig. 8 (a), with a $1^{\circ} \times 1^{\mathrm{o}}$ received power image of each $\mathrm{CC}$ taken with an angular step size of $0.05^{\circ}$, Fig. 8 (b). The two-way pattern is a 2D Gaussian
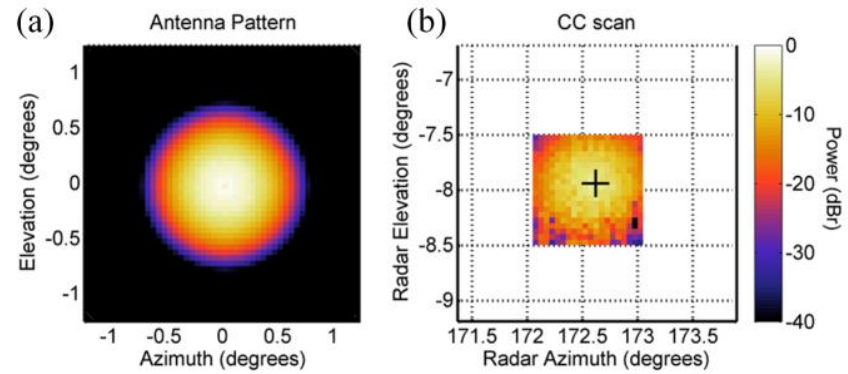

Fig. 8. Determining the direction to a $\mathrm{CC}$ reflector in the radar frame of reference showing (a) a $2 \mathrm{D}$ Gaussian fit to the laboratory measured AVTIS antenna pattern, and (b) a $1^{\circ} \times 1^{\circ}$ raster scan of a field $\mathrm{CC}$ at $1.86 \mathrm{~km}$ range. The cross marks the center of correlation between the antenna pattern and the CC scan. Correlations of 0.99 are typical in clear weather, falling to around 0.9 for poor visibility.

fit to a laboratory measured antenna pattern interpolated to $0.005^{\circ}$, setting the precision to which the azimuth $\left(\theta_{c c}\right)$ and elevation $\left(\phi_{c c}\right)$ gimbal angles are measured. The correlation is usually high $(>0.99)$ in clear weather even at long range although inclement weather adds noise and reduces the correlation to about 0.9 . Scans with correlations below 0.5 are discarded since they correspond to conditions of heavy rain during which the CCs are obscured.

Having recorded $\theta_{c c}$ and $\phi_{c c}$ for each $\mathrm{CC}$ in a particular occupation, the origin of the radar frame of reference is located at the AVTIS dGPS position and rotated about that origin to align the radar measured $\mathrm{CC}$ positions with their respective dGPS coordinates. Determining a complete $3 \mathrm{D}$ transformation in principle requires a minimum of 2 reference $\mathrm{CCs}$ to constrain the instrument orientation in terms of rotation about the $x, y$ and $z$ axes

However, it is often impractical to have three CCs in the field due to inaccessibility of terrain around the flanks of the volcano. Thus we tried to reduce the degrees of freedom by leveling the gimbal at each occupation setup. A perfectly level gimbal removes the need to determine the rotation of the radar frame of reference around the $x$ and $y$ axes leaving only the rotation around the $z$ axis. This is parameterized as an azimuthal offset, $\theta_{a d j}$, between local grid north and the zero azimuth axis of the AVTIS gimbal, the radar 'boresight'.

To measure $\theta_{a d j}$ we use a simple iterative routine to minimize the distance between the dGPS positions of the corner cubes, $C C_{G P S}(x, y, z)$ and the $\mathrm{CC}$ positions calculated using a spherical to cartesian transform of the form

$$
\begin{aligned}
& C C_{R A D A R}\left(\theta_{c c}+\theta_{a d j}, \phi_{c c}, R_{c c}\right) \\
& \rightarrow C C_{R A D A R}(x, y, z)+R A D_{L G}(x, y, z),
\end{aligned}
$$

where $C_{R A D A R}(\theta, \phi, R)$ is the radar angle and range vector to each reflector, $C_{R A D A R}(x, y, z)$ is the $x y z$ offset of the $\mathrm{CC}$ from the radar and $R A D_{L G}(x, y, z)$ is the position of the radar in local grid coordinates (determined by dGPS). Here we use the dGPS-measured range $R_{c c}$ between the radar and CCs since the range precision is far higher $(\mathrm{cm})$ than the radar range measurement $(0.85 \mathrm{~m})$.

Using $\theta_{a d j}$ as the iteration variable typically matches any particular $\mathrm{CC}$ position to within $1 \mathrm{~cm}$ between the two 
(a)

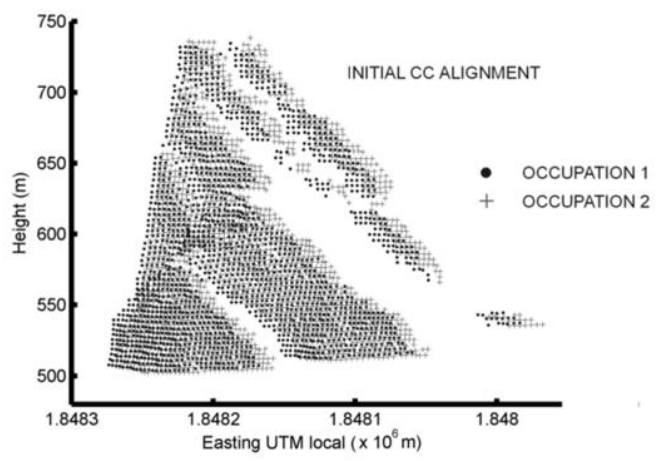

(b)

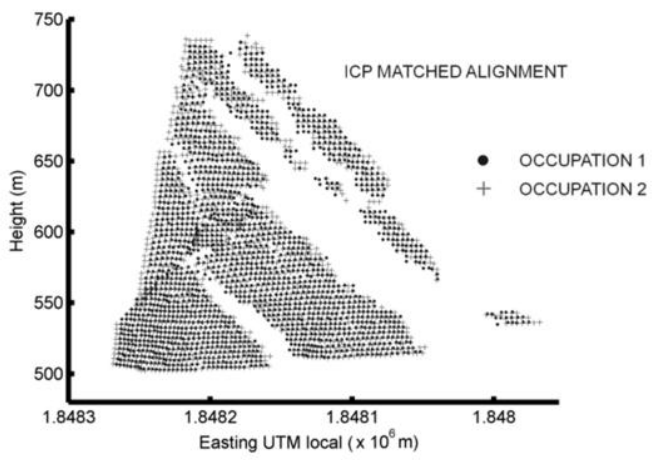

Fig. 9. (a) The use of CC reference reflectors and dGPS measurement did not align occupation to occupation data with sufficient accuracy, and (b) static terrain common to each occupation is matched using an Iterative Closest Point (ICP) algorithm to produce a final translation and rotation which is then applied to the entire DEM. The data shown is for the $2 \mathrm{~km}$ range terrain shown in Fig. 7.

reference frames, however the value of $\theta_{a d j}$ can vary from CC to $\mathrm{CC}$ by up to $\sim 0.2^{\circ}$ within an occupation. This is most likely a consequence of the precision to which the gimbal could be leveled and corresponds to a maximum absolute error in DEM position of $\sim 3.5 \mathrm{~m} / \mathrm{km}$ from the radar. To help account for this, we add an analogous term in elevation $\left(\phi_{a d j}\right)$ to the minimization technique to constrain the instrument orientation further.

\section{1) Post-processing: Iterative minimization of DEM to known static topography}

Analysis of field data shows that the standard deviation of $\theta_{c c}$ and $\phi_{c c}$ ranged between $0.02^{\circ}$ and $0.05^{\circ}$ for each occupation. To minimize error each CC was scanned as many times as was practical and the average angular values used. Despite this, a small but significant offset between occupation DEMs is still evident particularly at the edges of terrain, Fig. 9 (a). Thus a final rotation and translation for each DEM is calculated using an iterative closest point (ICP) algorithm to match surfaces from different occupations, Fig. 9 (b). Since the volcanic terrain can actually change between occupations, we select a piece of topography common to each occupation and known to be static for use as an absolute reference. For cases where there is no known reference topography, the occupation with the largest number of CC scans is used to generate the reference surface.

\section{2) Post-processing: Final DEM interpolation}

The final stage of DEM generation is to interpolate the

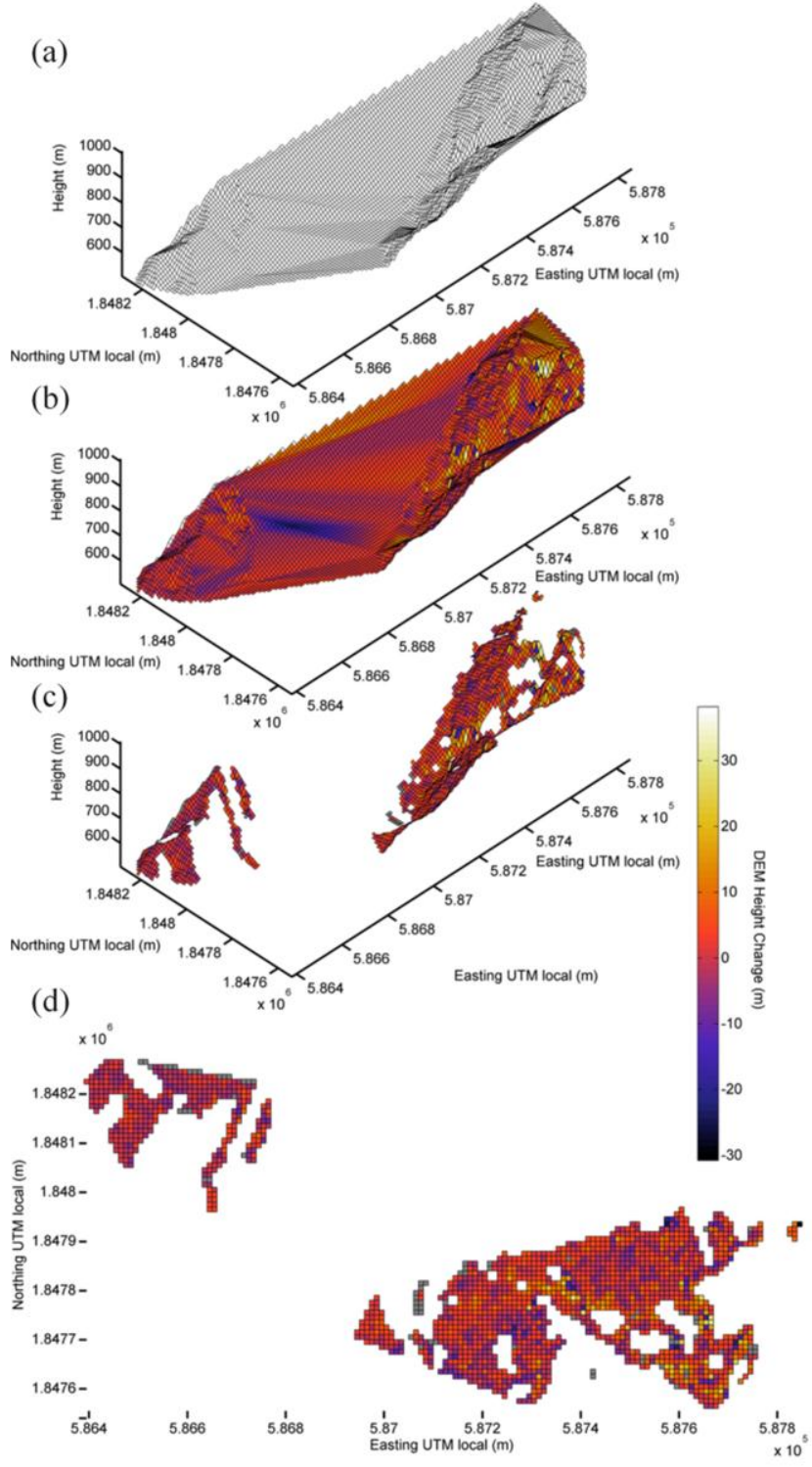

Fig. 10. (a) Interpolation onto the regular GPS grid produces occlusion surfaces linking disconnected topography. (b) Comparing DEM height differences between different scans produces significant errors. (c) Surface mask using the distance from the $x y$ coordinates of the interpolation grid to $x y$ positions in the original point data as a threshold criterion. (d) The "top down' view of the same surface shows that significant areas of localized topography are occluded from the radar viewpoint. The topography shown is the same as plotted in Fig. 7.

irregularly spaced point cloud surface data onto regular east and north postings. The data points produced by AVTIS in the previous steps are in geographic coordinates, but are inherently irregular in east and north spacing due to the translation from a spherical coordinate system. To compare data between occupations requires interpolation of data height $z$ onto a laterally coincident grid. Strictly speaking the spacing of the interpolation grid should be based on a combination of the range to scene and the angular beamwidth of the radar, with close range topography requiring a finer grid than long range topography. For most data this is computationally awkward and we use a regular cartesian grid with resolution selected on a case-by-case basis. Many algorithms and 
software packages exist to perform 3D interpolation, each with its own advantages and disadvantages; we use a linear interpolation between the data points; however the most significant problem with interpolation of AVTIS data is the effect of shadowing due to occlusion of the radar LOS by intervening topography. A relatively small change in topography can obstruct the view of more distant terrain producing a large surface that is an artifact of the radar point of view.

In some cases this effect is very obvious (e.g. the large apparent surface linking the foreground ridge and dome in Fig. 10 (a) and (b), and is relatively easy to resolve by disregarding any topography outside of a large $x y$ region of interest. However, one must also account for occlusions on a smaller scale within the target topography itself, Fig. 10 (c).

To remove these artifacts we apply a mask to the data determined by the distance from each location in the regularized $x y$ interpolation grid to the $x y$ positions of the point data (ignoring $z$ ), Fig. 10 (d). Interpolated values that lie too far from the point data are removed from the surface. After qualitative analysis of field data, the minimum distance criterion has been chosen to be one third of the $3 \mathrm{~dB}$ radar beamwidth at the maximum radar range contained within the DEM dataset. Reducing this distance criterion produces heavily perforated interpolated surfaces whilst increasing the criterion fails to sufficiently remove the false surface artifacts. Having removed these artifacts, the DEMs can finally be compared between occupations, Fig. 11. Analysis of DEM comparison is given in section VII.

\section{LONG RANGE PERFORMANCE}

On occasion, AVTIS has been located at long range vantage points, near the limit of radar signal-to-noise ratio (SNR). For ranges greater than $\sim 4 \mathrm{~km}$, the magnitude of radar returns is comparable to interference peaks produced by the stepper motors which control the gimbal, even when it is stationary. By reducing the current drawn by the gimbal motors to hold the instrument head in position this problem can be minimized. Whilst this works in calm conditions it does make the system subject to externally induced movement of the gimbal, particularly buffeting by wind, which can introduce errors into the absolute knowledge of the gimbal angles. Windy weather produces significant interference since the motors draw more current to maintain position.

At long range, this interference can dominate the spectrum with discrete, slowly changing peaks which appear at a quasiconstant range across a single axis scan. If the interference is not removed then the DEM is corrupted by a static 'wall' of false returns when assessing the range to maximum signal return, Fig. 12 (a).

Algorithms have been developed to remove this interference in post-processing by comparing the radar spectra over several successive LOS. Compared to the spread returns from natural terrain, the interference is discrete in frequency and static over the time scale of several LOS. Averaging a number of consecutive LOS spectra to improve SNR, gimbal interference lines are identified as peaks with edges that exceed a filtered-
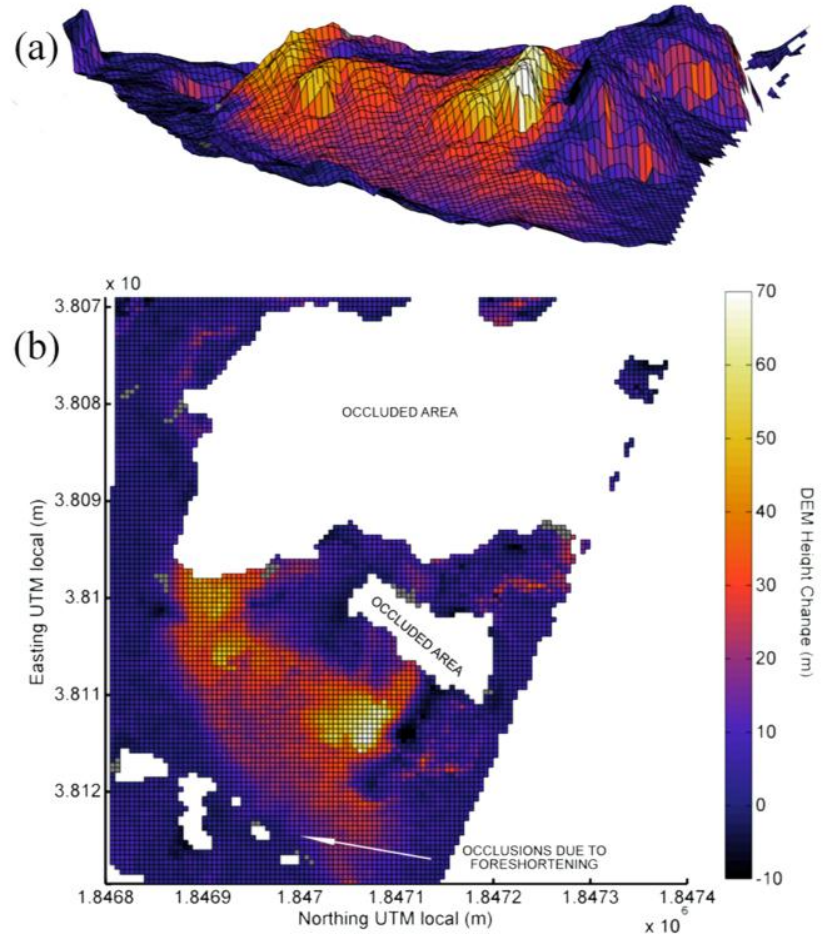

Fig. 11. Close range occlusion effects. (a) DEM surface orientated to approximately match the radar point of view. This DEM shows 10 days of growth of the Montserrat lava dome in Nov, 2005. The range to topography was 700 to $1600 \mathrm{~m}$. (b) The 'top down' view shows significant occlusion with most of the rear of the crater obscured by the dome along with a large section at the top rear of the dome. Note also that several regions at close range are occluded due to foreshortening

gradient tolerance and are subsequently subtracted from the spectra. This results in a visibly significant improvement in single axis scans and extracted surface fidelity, however the low SNR still produces a DEM with a relatively poorly defined surface, Fig. 12 (b).

The best method we have found to improve DEM quality is to average the LOS spectra over successive scans to reduce noise. This requires calculation of the time that each individual LOS was captured so that the spectra can be reinterpolated onto regularized range spectra according to the $\mathrm{CC}$ range drift calibration and is a not insignificant task. However, even a moderate number of averages greatly improve data fidelity, Fig. 12 (c). The penalty is that, for target ranges greater than $\sim 4 \mathrm{~km}$, a useful DEM can only be generated on a day-to-day timescale rather than hour-by-hour, but this is still on a sufficiently short timescale to measure temporal changes in the lava dome.

\section{RADAR CHARACTERIZATION}

Principally, the AVTIS radar measures terrain topography through rastered measurements of the scene. However AVTIS can also be considered as a clutter imaging radar allowing one to extract the Radar Cross Section (RCS), $\sigma$, of the target terrain. RCS quantifies the ratio of backscattered power relative to the power incident at the target of interest. In practice, the RCS of target terrain depends on a large number of poorly known factors: size, shape, material reflectivity, 

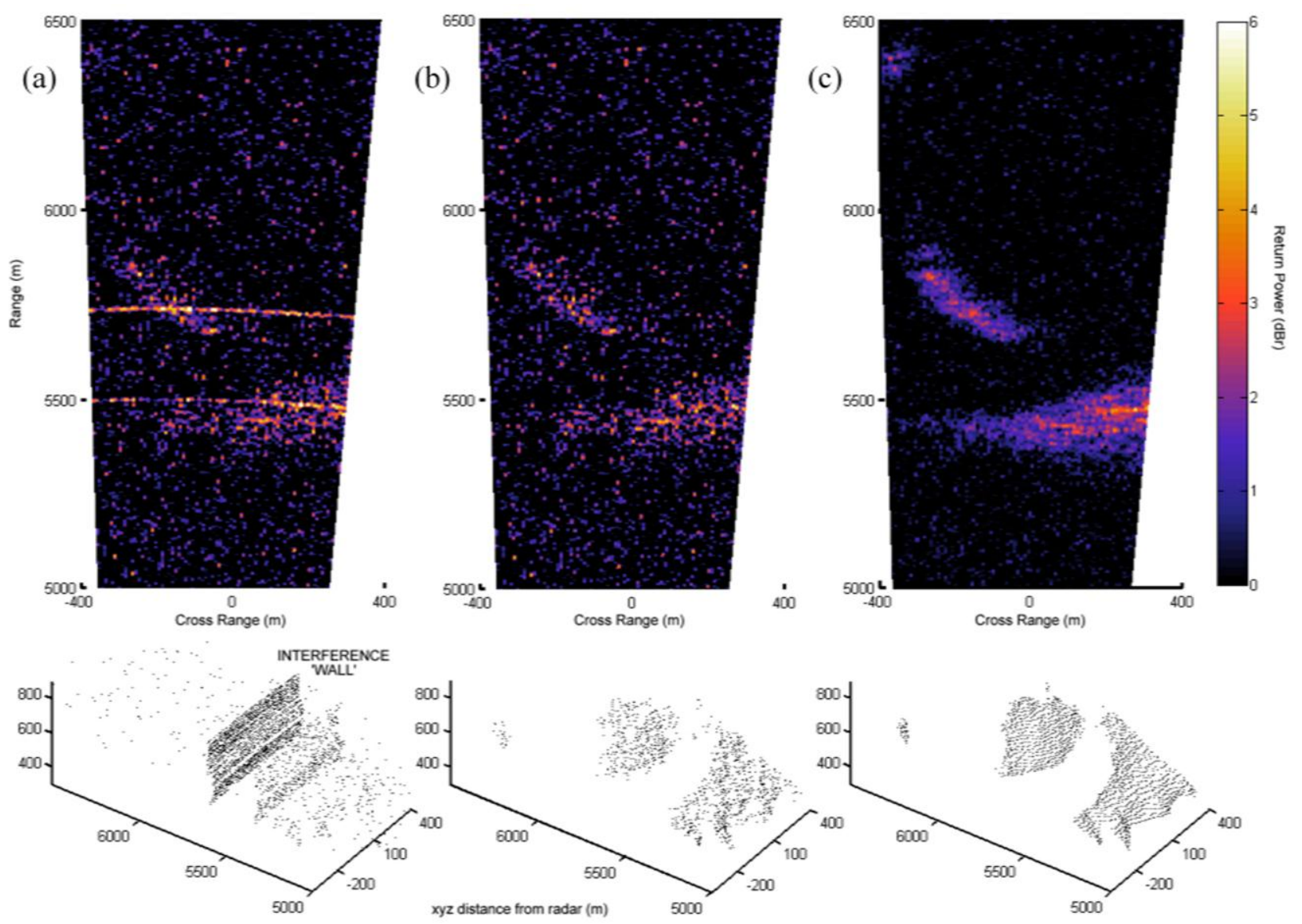

Fig. 12. Long range imaging of the lava dome. Plan Position Indicator (PPI) display of a single elevation and extracted 3D surface for (a) a single scan, (b) a single scan with interference removed, and (c) averaged surface from 11 successive scans. At ranges greater than $\sim 4000 \mathrm{~m}$ discrete electronic interference can be a serious problem completely masking the target topography and must be removed. Even without interference the low SNR produces a poor quality surface. Averaging of spectra from a number of successive scans are required to significantly improve PPI and surface fidelity.

polarization, surface roughness and incident geometry of the incident radar beam. However, one can account for the fading of returns with distance and atmospheric losses using the wellknown radar range equation.

The power recorded at the radar receiver for a point target at range $R$ is

$$
P_{R E C}(R)=\frac{P_{t} G_{a n t}^{2} \lambda^{2}}{(4 \pi)^{3} R^{4}} \cdot \sigma \cdot \frac{G_{R E C}(R)}{L_{\alpha}(R)},
$$

where $P_{t}$ is the power transmitted, $G_{\text {ant }}$ the antenna gain, $\lambda$ the carrier wavelength, $R$ the range to target, $G_{R E C}$ the receiver gain and $L_{\alpha}$ the atmospheric loss [17]. These last two terms are range (or equivalently IF frequency) dependant. Atmospheric loss can vary significantly at $94 \mathrm{GHz}$ from $0.35 \mathrm{~dB} / \mathrm{km}$ (clear dry air) up to $2.5 \mathrm{~dB} / \mathrm{km}$ depending upon humidity and weather [18]. Rainfall will significantly increase losses up to the point that the target terrain is obscured. The receiver gain $G_{R E C}$ consists of losses in the front end (waveguide, circulator and mixer) and gains due to IF amplification (Fig. 5).

Calibration of the AVTIS range (frequency) gain profile has been confirmed by modeling two different aspects of system performance: the return power from $\mathrm{CC}$ reflectors as a function of range and the received spectrum noise floor.
Returns were recorded over the course of two weeks from 225 separate scans of 6 different $\mathrm{CC}$ reflectors deployed on Montserrat. Each $\mathrm{CC}$ had a nominal $\sigma$ of $+20 \mathrm{dBsm}$ although this is a maximum possible value due to limitations in accurately aligning the CCs in the field. The returns from the CCs also varied significantly with the weather so only the maximum reflected power values recorded during the two week period were used with equation (3) to give an estimated minimum one-way atmospheric loss of $1.3 \mathrm{~dB} / \mathrm{km}$ for Montserrat, Fig. 13. Agreement between theory and data was to within $\pm 1.5 \mathrm{~dB}$ at ranges from $0.4-1.9 \mathrm{~km}$.

The system noise floor was calculated using the receiver gain profile combined with an estimate of system noise as a function of frequency. The estimated receiver noise figure of $10 \mathrm{~dB}$ corresponds to a receiver input noise power spectral density (NPSD) of $-164 \mathrm{dBm} / \mathrm{Hz}$, or $-149 \mathrm{dBm}$ for each 30.5 $\mathrm{Hz}$ FFT bin. However, the noise level is further increased by transmitter AM noise coupling via the transmit-receive leakage which is around $-35 \mathrm{~dB}$, corresponding to a leakage power of $-14.5 \mathrm{dBm}$. The quoted $\mathrm{AM}$ noise level of the transmit power amplifier is $-130 \mathrm{dBc} / \mathrm{Hz}$ and is assumed to be spectrally flat. This implies that an input AM NPSD of -144.5 $\mathrm{dBm} / \mathrm{Hz}(-130 \mathrm{dBm}$ per FFT bin) is delivered to the receiver 


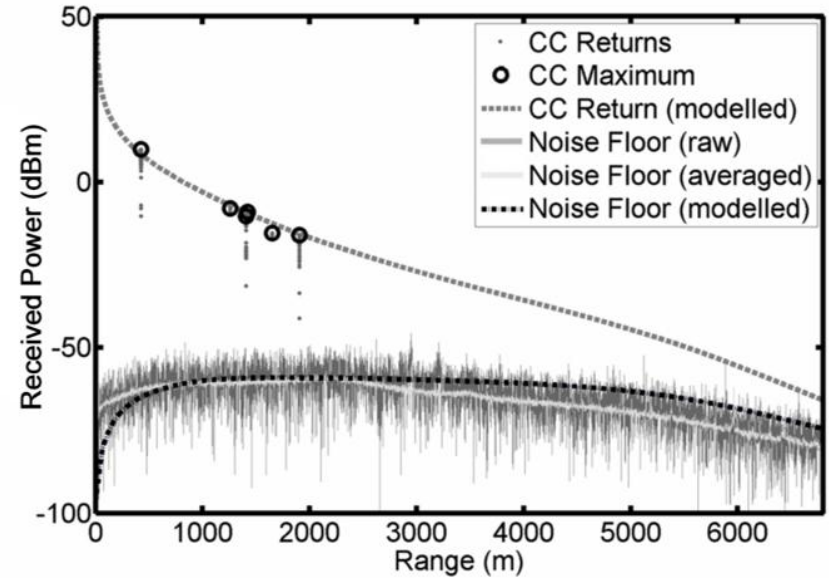

Fig. 13. Data and models characterizing AVTIS radar performance. Maximum reflected power from 6 fixed $\mathrm{CC}$ retro reflectors recorded in the field on Montserrat over a period of two weeks gave a minimum one way atmospheric loss of $1.3 \mathrm{~dB} / \mathrm{km}$. Noise floor data are from clear sky measurements and show good agreement with models based on component specification and laboratory characterization

chain which clearly dominates over the receiver noise. The noise floor plotted in Fig. 13 is the combination of both these contributions amplified by the receiver gain profile. The discrepancy between theory and measured spectra at the low and high ends of the spectrum is thought to be due to the leakage and the AM noise levels not being spectrally flat. Nevertheless, this calculation shows good agreement with the data and this characterization is applied to data gathered from topographic maps to give an estimate of terrain RCS.

\section{TERRAIN RADAR CROSS SECTION}

Analysis of power reflected from extended terrain is more complicated than for a point like target since the amount of backscattered energy depends upon the area of the beam incident on the terrain which, in turn, depends on the surface inclination and geometry.

At one extreme, (3) shows that returns from a point scatterer reflect isotropically and power at the receiver falls off proportional to $1 / R^{4}$. Alternatively, for a beam filling target at normal incidence, all of the incident power is reflected back to the radar and leads to a fall-off proportional to $1 / R^{2}$. For inclined terrain the situation is more complex and the actual fall-off with range lies somewhere between these extremes. The geometry for a typical AVTIS setup is shown in Fig. 14.

The antenna elevation angle, $\phi$, typically varies between $5^{\circ}$ and $20^{\circ}$ looking up at volcanic terrain. The terrain slope, $\varphi$, usually has a value somewhere between $20^{\circ}$ and $70^{\circ}$ although any angle is possible. This gives a typical range of grazing angle, $\delta$, from $0^{\circ}$ to $70^{\circ}$. Here the situation is analogous to the generalized radar range equation for the range bin-limited case in which the received power falls off proportional to $1 / R^{3}:[17]$

$$
P_{R E C}=\frac{P_{t} G_{a n t}^{2} \lambda^{2} G_{R E C}(R)}{(4 \pi)^{3} R^{3} L_{\alpha}(R)} \cdot \sigma^{0} \cdot \frac{\Delta R \theta_{3 d B}}{\cos \delta} .
$$

For extended terrain the quantity of interest is $\sigma^{0}$, the normalized RCS of the terrain which is the RCS per unit area.

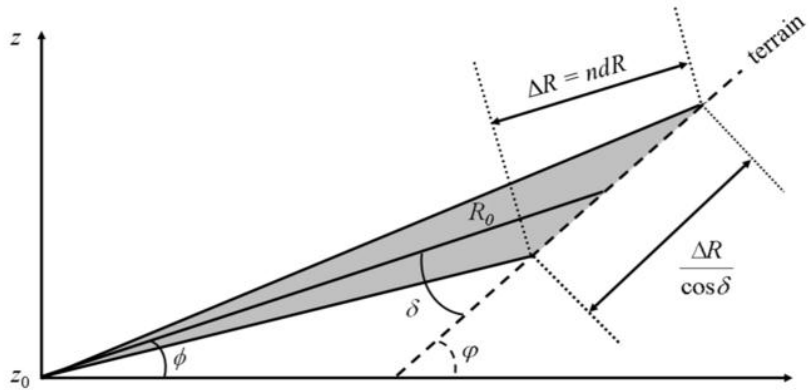

Fig. 14. Geometry of incident radar beam on terrain. The radar is located at $z_{0}$ and is usually looking up at an elevation angle $\phi$ onto terrain which itself has a slope angle $\varphi$. Application of the low pass filter in DEM extraction sets an effective footprint size $\Delta R$ which is $n$ times (integer number of range bins) larger than the spectral resolution of $d R$. The actual beam intercept on the terrain then depends on the grazing angle $\delta$.

This particular form of the radar equation assumes that the incident beam is limited in azimuth by the $3 \mathrm{~dB}$ width of the antenna pattern, $\theta_{3 \mathrm{~dB}}$, and has an incident footprint set by $\delta$ and the range bin extent $\Delta R$. We have substituted this as $\Delta R=$ $n d R$, where $n$ is the number of range bins filtered in the DEM extraction and $d R$ is the range bin size defined by the FFT. Using the same values of atmospheric loss and system gain as for the CC modeling in Section V, we corrected DEM data raw power reflectivity values using (4) and compared the values within a band of $20^{\circ}<\delta<70^{\circ}$, Fig. 15 .

This comparison suggests that $\sigma^{0} \sim-18 \mathrm{~dB}$ gives the best general agreement with the model as the mean value for volcanic terrain at these incidence angles. The data show a similar spread to that predicted by the model. Data that fall below the modeled curves (e.g. at $2200 \mathrm{~m}$ ) relate to returns at very shallow grazing angles where the model starts to break down.

There are very few published values of $\sigma^{0}$ at $94 \mathrm{GHz}$ for bare surfaces and none for volcanic terrain to our knowledge. Currie et al [19] shows measurement of several terrain types suggesting a range of approximately $-24 \mathrm{~dB}<\sigma^{0}<-16 \mathrm{~dB}$. More recently, Ulaby et al [20] published a near grazing incidence $\left(<20^{\circ}\right)$ set of data for a collection of bare surfaces (gravel, asphalt, concrete, fields) which gives a range of -23 $\mathrm{dB}<\sigma^{0}<-9 \mathrm{~dB}$ for $\delta=20^{\circ}$. This suggests that $\sigma^{0} \sim-18 \mathrm{~dB}$ for volcanic terrain is broadly in line with other bare surface types.

Finally, having confirmed the form of range dependency we can correct scene data from received power to $\sigma^{0}$, Fig. 16 . The raw received power image, Fig. 16 (b), is corrected to $\sigma^{0}$, Fig. 16 (c), assuming an average $\delta$ of $45^{\circ}$. It is evident that the range dependency is suppressed and variations in terrain reflectivity become apparent. Terrain edges give a lower value of $\sigma^{0}$ due to the shallow grazing angle. In principle, the DEM shape could be used to calculate the local angle of incidence and use this to give a better local estimate of $\sigma^{0}$, however this has not yet been attempted.

Histograms of the raw received power and $\sigma^{0}$ images of Fig. 16 (b) and (c) are shown in Fig. 17 (a) and (b) respectively. These data were recorded on the clearest day encountered, 


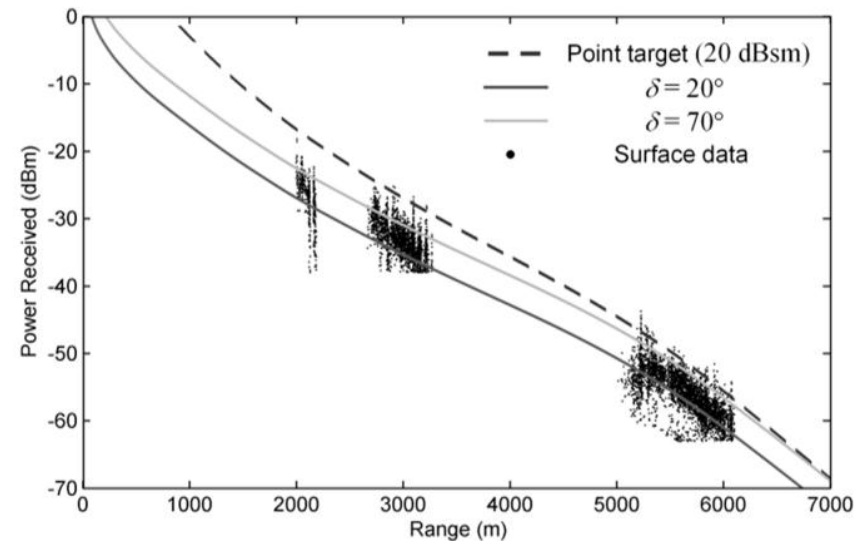

Fig. 15. Modelling of received power as a function of range versus field recorded data. The modeled power received assumes a fixed terrain $\sigma^{0}$ of -18 $\mathrm{dB}$. The surface data points are taken from several different views of terrain around the Soufrière Hills lava dome at three different ranges. The data are bounded by the model for grazing angles varying from $20^{\circ}$ to $70^{\circ}$. The 20 $\mathrm{dBsm}$ point target curve represents our standard CC calibration targets, showing that $\mathrm{CC}$ signal to clutter level makes CC location impractical beyond $\sim 2500 \mathrm{~m}$.

with the lowest atmospheric attenuation. The received power distributions corresponding to terrain at different ranges merge to reveal a single distribution in $\sigma^{0}$ indicative of the terrain type. From these we set a $\sigma^{0}$ threshold below which returns are discarded when generating a DEM surface. Whilst received power can be used to separate terrain from sky returns, the distinction is strengthened by considering $\sigma^{0}$.

\section{EXAMPLES OF DEM COMPARISON}

The primary aim of AVTIS is to record the change in topography produced by volcanic activity. From that, with assumptions, we can infer the volumetric rate of lava extrusion at a volcanic vent (typically a few $\mathrm{m}^{3} \cdot \mathrm{s}^{-1}$ ). This is simply the volume change between two DEM surfaces divided by the time interval between DEM acquisitions. The total volume change is the change in height for each DEM $x y$ grid point multiplied by the area set by the $x y$ grid spacing, summed over all $z$ points coincident between that pair of DEMs. The recorded time of each DEM is taken to be midway between the start and end of each data gathering occupation. Note that the extrusion rates calculated below only give the rate for the faces of the dome visible from the AVTIS point of view. The total extrusion rate of the dome can only be calculated using multiple viewpoints from locations all around the dome (which is usually impractical) or by making assumptions about the nature of dome growth in the unseen regions of topography.

AVTIS was deployed on Montserrat during early April of 2006 to record the lava extrusion rate as the growing dome became visible above the crater left behind by the last major dome collapse on 12 July 2003 (a photograph of the dome at the time of the deployment is shown in Fig. 20 (a)). During this deployment, dome growth was evident through visual observations, occurring predominantly on the eastern side of the dome. A few small rockfalls also occurred, removing material from the dome. AVTIS occupations that successfully (a)
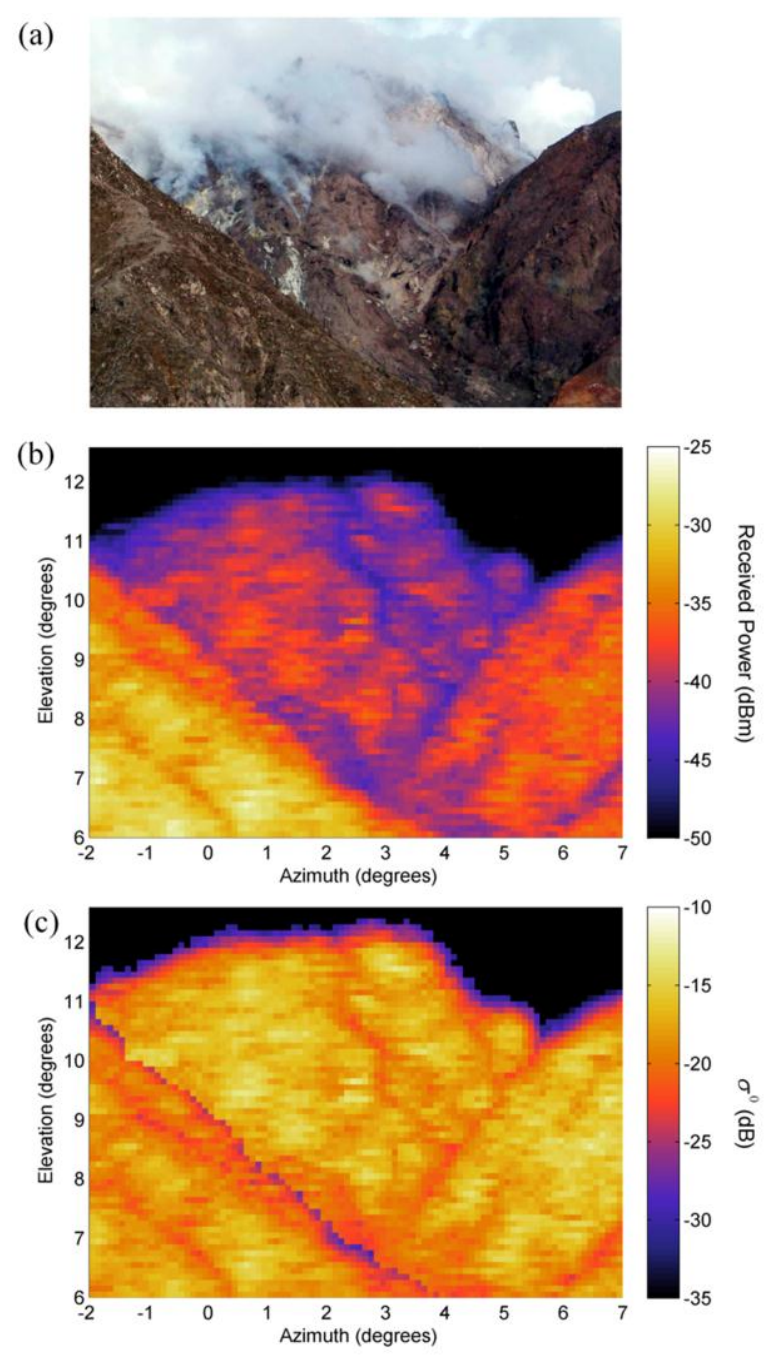

Fig. 16. Lava dome images from St George's Hill, Montserrat, Sept. 2008. (a) Photograph of the scene. (b) Radar received power image. (c) Normalized RCS image. Color scales for (b) and (c) are based on the data histograms shown in Fig. 17 using the same $25 \mathrm{~dB}$ range to span the terrain data. The skyline is better defined in the RCS image.

measured dome growth were to the north east side of the dome: Bramble Airport (BA) at a range of $\sim 5500 \mathrm{~m}$ to the dome and Jack Boy Hill (JBH) at a range of $\sim 6000 \mathrm{~m}$. These two locations had a similar view of the dome but obscuration of the lower slopes of the dome by the old crater rim differed slightly (mainly due to the different elevations of the two sites). We typically scanned from BA during the day to get as close to the dome as was safe and JBH in the evening since access to BA was not possible after nightfall.

Here we compare three DEMs captured by AVTIS. The first DEM is an average of 4 scans captured on the afternoon of 31st March 2006 from BA. The second is an average of 3 scans captured from BA on the afternoon of 6th April 2006. The third DEM was an average of 7 scans captured from JBH a few hours later on the evening of 6th April. Visual observations suggested that there were no significant changes in the dome on that day.

The first comparison, Fig. 18 (a), shows the change between the first and second DEMs recorded from BA. The surface 

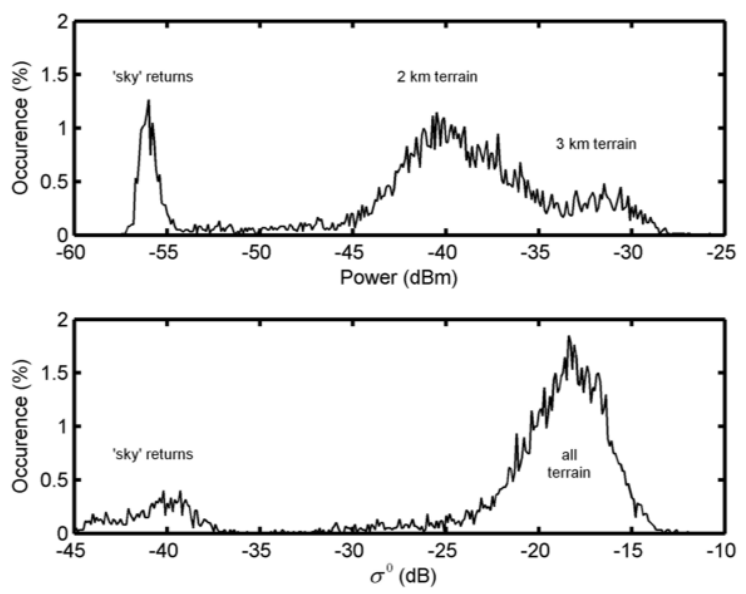

Fig. 17. Histograms of the data for Fig. 16 showing occurrence of (a) radar received power, and (b) normalized radar cross section, $\sigma^{0}$. The long tails to the left of the peaks of the terrain distributions represent returns from low grazing angles. The correction to RCS enhances the distinction between these low RCS value returns for terrain and the unwanted 'sky' returns (which represent the system noise floor).

plotted is the latter surface of the two on a grid spacing of $10 \mathrm{~m}$ with the color scale showing the height change between the two DEMs. Bulk growth on the east side of the dome is clearly measured. Note also the small collapsed section in the middle of the dome, showing here as a dark region corresponding to a reduction in dome height. The grey region to the south of the collapse represents topography that was obscured at the time of the first DEM aquisition before the collapse. Topographic regions such as this that are non-coincident between DEMs are ignored when calculating height difference between DEMs. In this case the occlusions were primarily caused by the changing shape of the lava dome.

A gap of a few hundred meters between the lava dome and crater provided a natural division in the topography giving two distinct DEM sections, one for the dome and one for the crater. The extrusion rate was calculated by considering the dome DEM section only. The crater DEM section was assumed to be essentially unchanged during the field deployment and was used as the reference static topography to co-locate the DEMs (as described in Section III, B, 11). In this case, the calculation gives an 'apparent' extrusion rate, because rockfall from the lava dome was accumulating downslope in the occluded area between the dome and the crater wall. A fuller discussion of this is given in [14].

The total volume change between the dome DEM sections in these data was $1.605 \times 10^{6} \mathrm{~m}^{3}$ (a mean increase in height of $13.66 \mathrm{~m}$ over an area of $117500 \mathrm{~m}^{2}$ ). To evaluate the accuracy of this measurement we analyzed the distribution of height difference for both the dome and crater sections. Fig. 18 (b) shows the histograms of height change for each section, binned at $1 \mathrm{~m}$ intervals. The distribution for the crater section is evidently not Gaussian and we found that both sections are fitted well by a Laplacian distribution (i.e. showing an exponential fall off either side of the mean). Using the Laplacian fit we calculated a mean height change of $0.62 \mathrm{~m}$ for the crater with a standard deviation of $4.65 \mathrm{~m}$. Using this as a measure of the DEM surface change accuracy, the

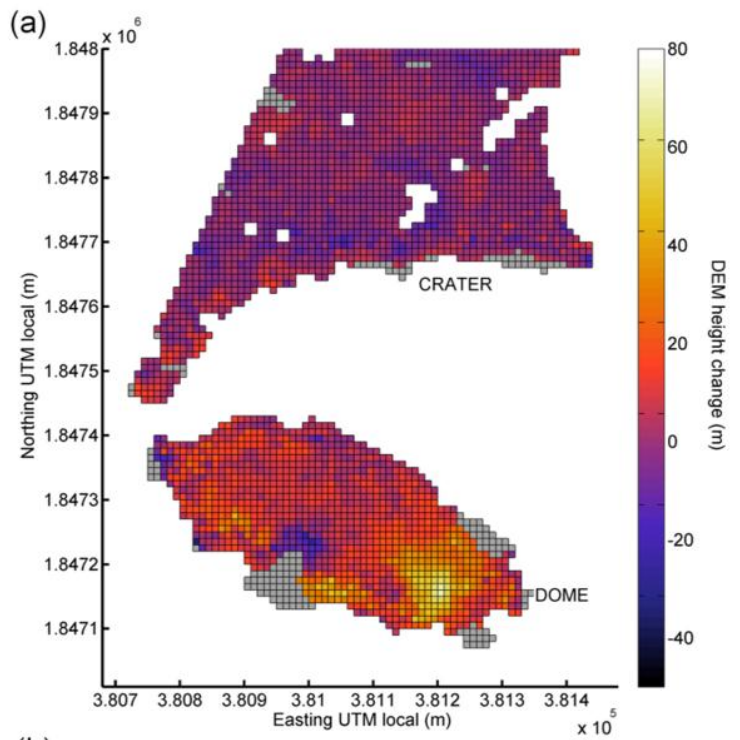

(b)

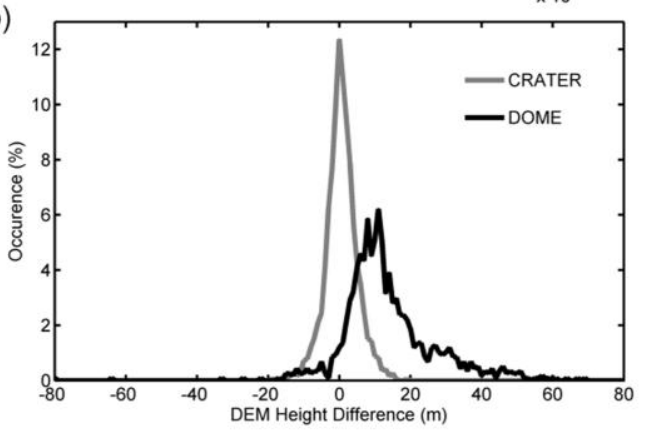

Fig. 18. (a) DEM recorded at a range of $\sim 5500 \mathrm{~m}$ showing dome growth over 6 days measured from the same location, Bramble Airport, Apr. 2006. Regions colored grey indicate non-coincident topography between the two DEMs. (b) Histogram of height difference binned at $1 \mathrm{~m}$ intervals for the crater and dome regions separately.

standard deviation in the dome growth was calculated as 5.20 $\times 105 \mathrm{~m}^{3}\left(4.65 \mathrm{~m} \times 117500 \mathrm{~m}^{2}\right)$. Thus the volume change of the dome was constrained as $1.6 \pm 0.6 \times 10^{6} \mathrm{~m}^{3}$. The apparent extrusion rate is then simply the volume change divided by the time between scans. The acquisition time for each DEM was 3 to 4 hours giving an error of $\sim 3 \%$ over the 6 day interval, much less significant than the volume difference error. The final estimate of apparent extrusion rate for these data was calculated as $3.09 \pm 1.05 \mathrm{~m}^{3} \cdot \mathrm{s}^{-1}$.

The second example, Fig. 19 (a), shows the comparison of the first BA DEM with the JBH DEM. Here the volume change between the dome sections was $2.544 \times 10^{6} \mathrm{~m}^{3}$ (a mean increase in height of $27.68 \mathrm{~m}$ over an area of $91900 \mathrm{~m}^{2}$ ). The histogram of height difference for the crater section, Fig. 19 (b), is still fitted well by a Laplacian distribution, albeit a more spread distribution with no obvious 'zero' peak. The mean height change was $0.30 \mathrm{~m}$ and the standard deviation was $10.02 \mathrm{~m}$. We assume that this larger spread is a consequence of the relatively large radar footprint on the dome at long ranges (at a range of $6000 \mathrm{~m}$ the two-way $3 \mathrm{~dB}$ diameter at normal incidence is $52 \mathrm{~m}$ ). The ranging measurement filters the received radar spectra to give a measure of the average position of the incident beam upon the 


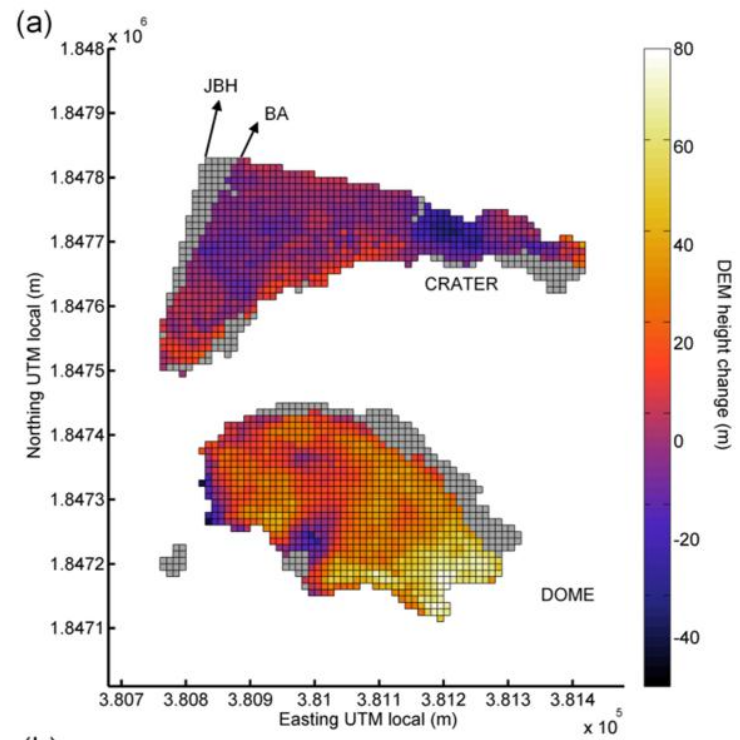

(b)

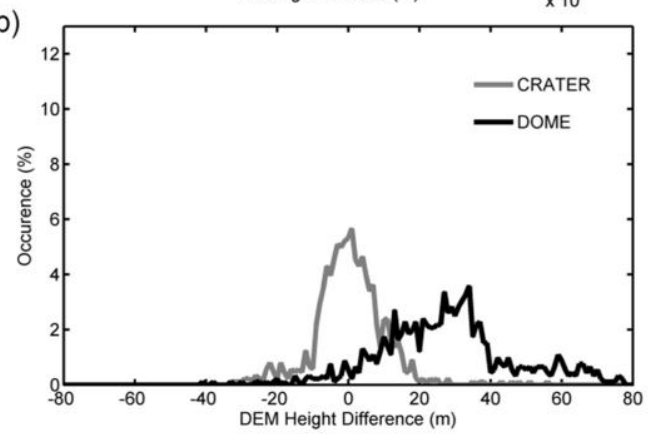

Fig. 19. (a) DEM recorded at a range of $\sim 6000 \mathrm{~m}$ showing dome growth over 6 days measured from two different locations, Bramble Airport and Jack Boy Hill, April 2006. (b) Histogram of height difference binned at $1 \mathrm{~m}$ intervals for the crater and dome regions separately. Topography from two different viewpoints degrades the surface correlation and increases errors in volume change measurement. Note that the scales for both graphs are the same as those in Fig. 18.

topographic surface (section III, B, 6) and will not be precisely the same for any given topography when measured from different occupation sites. It is also possible that the absolute registration of the DEMs for each occupation differs slightly introducing an additional error.

Despite these issues, following the analysis described for the first example, the volume change for the differing viewpoints was calculated as $2.54 \pm 0.92 \times 10^{6} \mathrm{~m}^{3}$ with a corresponding apparent extrusion rate of $4.75 \pm 1.72 \mathrm{~m}^{3} \cdot \mathrm{s}^{-1}$. This value is close to that from the first DEM comparison, to within the stated errors, showing that DEMs from different locations can be compared, although we believe that it is preferable to measure dome growth from the same field location when possible.

At ranges of $\sim 6000 \mathrm{~m}$, these surfaces were recorded near the limit of AVTIS's capability (fig. 13). Consequently the errors calculated above represent the worst case in uncertainty. Extrusion rates derived from terrain measurements at closer ranges would have lower error bounds. In addition, at all ranges localized topographic change on the dome is clearly discernible yielding valuable information not readily obtainable by alternative means. We note that no other lava flux data were available during these studies, which may have been used for cross-validation of our results.

\section{RADIOMETRIC RESULTS AND ANALYSIS}

The secondary aim of AVTIS is to provide MMW radiometric thermal imagery of the lava dome in all weathers. This is achieved using the heterodyne radiometric mode with the radar transmit power switched off and the IF selected accordingly (Fig. 2). The dual active and passive measurement modes of AVTIS entail a more complicated front end design than a basic radiometer since the receiving antenna is shared with the radar mode and requires isolation between the transmit and receive paths. Loss in the circulator degrades the sensitivity of the radiometric receiver. Furthermore, direct leakage from the radar transmit arm also influences the radiometer performance.

The effective noise temperature of the front end has been observed to be extremely sensitive to changes in leakage from the radar transmitter signal despite the transmitter being attenuated by $55 \mathrm{~dB}$ during radiometric scans. Leakage varies significantly across the RF bandwidth and care must be taken to set the LO frequency at the center of a narrow band of minimal leakage such that radiometer sensitivity is maximized. This effect is sensitive enough that the system noise temperature at switch on is approximately 10 to $15 \%$ above normal before the LO centre frequency thermally stabilizes. In addition, the radiometer is also degraded by electronic interference in the system, primarily from the gimbal stepper motor pulses. Previous analysis has shown that the effective thermal sensitivity of the AVTIS radiometer in the field is only $5 \mathrm{~K}$ as opposed to the theoretical resolution of $2 \mathrm{~K}$ [15].

The AVTIS radiometer uses a two stage calibration process splitting receiver calibration into two sections, namely the front end (antenna, waveguide, transmit/receive circulator and mixer) and the IF amplification chain [16]. Short term gain fluctuations in the IF chain are calibrated using a two-level 'noise-adding' or 'noise-injection' calibration technique [21] for each LOS in a raster scan. Losses in the front end are stable over much longer timescales and are accounted for by periodic referencing to the thermal temperature gradient of the atmosphere [15].

The process for gathering radiometric imagery in the field is essentially identical to that described above for the radar. Thermal data is co-located in post-processing onto the topography measured by the radar mode. The only additional requirement is to acquire reference profiles of the sky which take about 1 minute. This is carried out at the end of each radiometric image acquisition.

Radiometric imagery has been gathered on two separate field campaigns on Montserrat. The bulk of these data were recorded at ranges typically up to $6 \mathrm{~km}$ where the $0.74^{\circ}$ beamwidth translates to an incident beam spot size of $73 \mathrm{~m}$ diameter on the mountain. This is sufficient to observe bulk properties of the dome, but insufficient to resolve any fine detail. A sample MMW image recorded at a range of $6 \mathrm{~km}$ is 
(a)

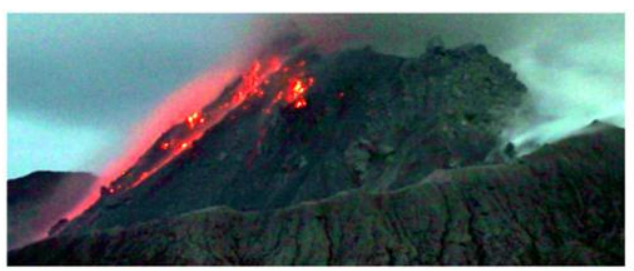

(b)
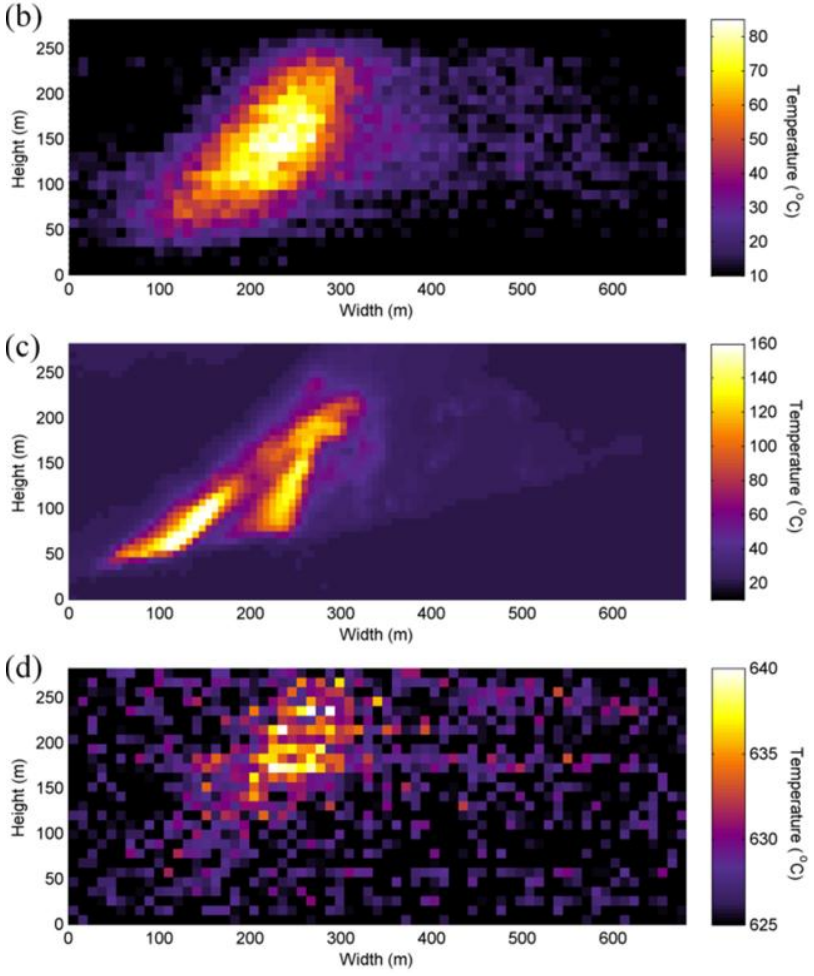

Fig. 20. (a) Visible, (b) MMW, and (c) IR images of the Soufrière Hills lava dome, Montserrat, April 2006. View from Jack Boy Hill at a range of $\sim 6000$ $\mathrm{m}$. Note that the images are not simultaneous but were taken under similar viewing conditions. (d) MMW image taken through heavy rain.

shown in Fig. 20 along with thermal IR and visible images for comparison. Although the MMW image lacks the detail of IR, the MMW image can easily be recorded through cloud and light drizzle. Even when there is heavy rain there remains some indication of the remnant heat from the dome that has not been scattered and/or absorbed by rain droplets. Note that the poorly calibrated temperature scale in Fig. 20 (d) is due to rain obscuring the reference sky profile.

Opportunities for high resolution MMW radiometric imaging of the Soufrière hills lava dome have been limited by the practicalities of reaching suitable safe vantage points. To date there has only been one successful close range $(1100 \mathrm{~m})$ acquisition on the 4th of November 2005.

AVTIS was deployed during the onset of new dome growth following and the only direct line of sight to the new dome available was from the Perche's Mountain site on the crater rim, accessible by helicopter. The slow acquisition time for a radiometric scan, combined with typically cloudy conditions on the mountainside meant that only one radiometric image of the dome was captured. From Perche's the dome had an angular size of $16^{\circ} \times 22^{\circ}$ and entailed a full 35 minutes to acquire a single rastered image of the dome at $0.25^{\circ}$ increments, shown in Fig. 21 (a).

Although calibration of the AVTIS radiometer had been carried out in the laboratory, the practical difficulty of simulating the elevated temperatures present in a volcanic scene meant that the accuracy of thermal imagery of the dome acquired with AVTIS was evaluated by comparison with simultaneous IR imagery captured with a FLIR Systems Thermacam S40 infrared camera. The S40 captured one image every ten seconds for the duration of the AVTIS scan. During this 35 minute period, rockfalls on the surface of the dome varied the thermal appearance of the dome such that any particular IR image was acquired at a time corresponding to a particular elevation in the MMW image. Here we present a qualitative imaging comparison between the two datasets by choosing a single IR image acquired approximately half way through the AVTIS raster scan, Fig. 21 (b).

The most obvious distinction between these particular datasets is that the IR temperature range is far greater than for the MMW, with peak temperatures of $350{ }^{\circ} \mathrm{C}$ compared to 220 ${ }^{\circ} \mathrm{C}$ respectively. Indeed throughout the entire IR dataset the maximum temperature recorded was $\sim 400{ }^{\circ} \mathrm{C}$. This difference is a consequence of the different angular resolutions of each imaging system, with IR able to resolve much finer detail than MMW. Angular resolution is a function of both wavelength and receiver aperture size. Here the handheld IR camera has 10-times better spatial resolution than AVTIS, with an angular resolution of approximately $0.075^{\circ}$ compared to the AVTIS $3 \mathrm{~dB}$ beamwidth of $0.74^{\circ}$.

To account for this difference and compare data directly, we therefore convolve the AVTIS antenna pattern with the IR data to yield a comparable, down-resolved IR image. The emissivity of volcanic rocks at MMW frequencies is unknown and was assumed to be similar to IR values for the purposes of this comparison. Using the 3D topography measured with AVTIS in its radar mode, this could in principle be achieved with a full 3D convolution of the extended beam profile of the AVTIS antenna. Such analysis, however, is non-trivial; we have instead reduced the analysis to a $2 \mathrm{D}$ image convolution. The image plane for both systems was taken to lie at a range of $1100 \mathrm{~m}$, the distance deemed to best represent the average range to the dome based on the radar topography. The IR data are corrected for atmospheric attenuation using a relative humidity of $80 \%$ and a range of $1100 \mathrm{~m}$ and then scaled to a height and width of $250 \mathrm{~m} \times 400 \mathrm{~m}$.

The angular one-way antenna pattern of $0.74^{\circ}$ is fitted to a 2D Gaussian distribution and translated onto the image plane, resulting in a $3 \mathrm{~dB}$ spot diameter of $14.2 \mathrm{~m}$ at $1100 \mathrm{~m}$. This is shown for scale next to the IR image inset in Fig. 21 (b). The resulting convolved IR image is shown in Fig. 21 (c). For comparison with the MMW image, these data are spatially resampled to match the AVTIS raster and plotted in Fig. 21 (d). The MMW, convolved IR, and resampled convolved IR data are plotted with identical temperature axes. Qualitatively, the images compare well, with most of the thermal features on the dome agreeing in amplitude and distribution. Two regions on the dome noticeably disagree: the lower left of the dome and the top right. In both of these regions the IR was 


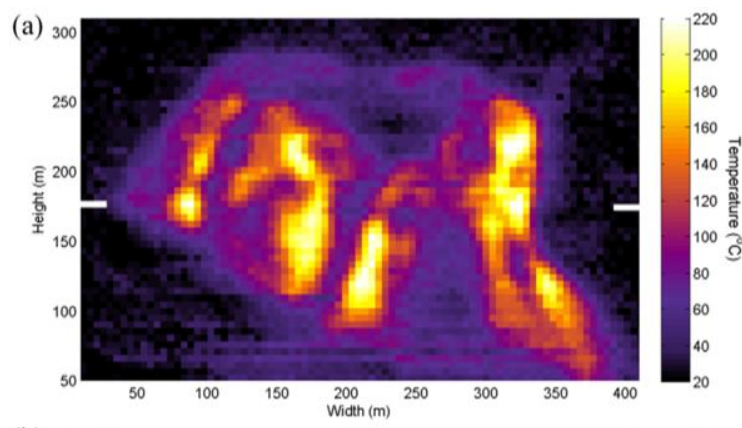

(e)
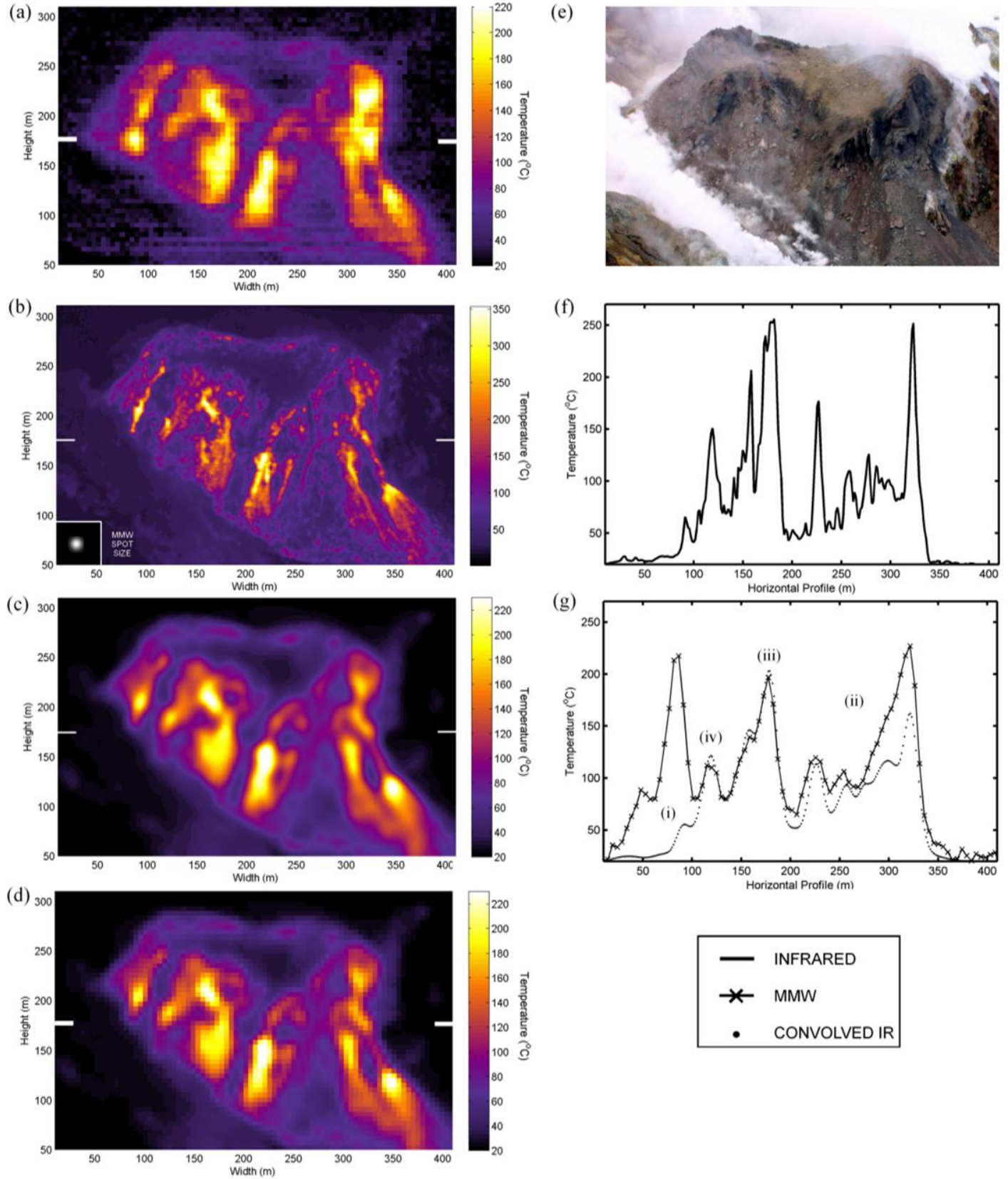

Fig. 12. (a) Rastered MMW radiometric image of the Soufriere Hills lava dome acquired over 45 minutes on 4th Nov 2005, from Perche's Mountain. (b) IR image taken midway through the MMW raster scan with AVTIS radiometric spot size inset. (c) Convolution of radiometric spot size with IR data. (d) Convolved data resampled to match MMW raster sampling. (e) Photograph of the lava dome during the raster scan. (f) Horizontal profile of the IR image taken at a height of $175 \mathrm{~m}$ as indicated by the white markers in (a) to (d). (g) Comparison of MMW and IR horizontal profiles at $175 \mathrm{~m}$. Points (i) to (iv) are described in Section VIII.

attenuated by gas and condensing steam emanating from the dome, clearly evident in the visible image, Fig. 21 (e). This is a good example of how thermal imaging of volcanoes with IR is clearly susceptible to obscurants, even during clear weather conditions.

Quantitative comparison between the data was carried out by selecting a particular elevation in the raster scan and examining the coincident IR image data at that same elevation. Each elevation took approximately $30 \mathrm{~s}$ for AVTIS to raster across, so there are three IR images for each of these horizontal profiles. One image was chosen with which to compare the horizontal profile for the MMW, IR and convolved IR. The horizontal profile data are plotted in Fig. 21 (f) and (g), with the corresponding elevation position marked on the imagery by white ticks in Fig. 21 (a) to (d).

Once again the finer structure visible in the IR is obvious compared to the MMW. Fig. 21 (g) has been labeled to show: (i) Attenuation due to condensing steam in the IR is almost total, with the dome appearing similar to the general background temperature of $25^{\circ} \mathrm{C}$ and a prominent thermal feature reduced from $220{ }^{\circ} \mathrm{C}$ (MMW) to $55^{\circ} \mathrm{C}$. (ii) Partial attenuation due to volcanic gas. Here the attenuation is less, but certainly sufficient to give a reduced temperature reading of the dome surface from the IR. (iii) Good agreement for a 
region of the dome that is not obscured to IR, especially given the relatively poor thermal resolution of AVTIS $(5 \mathrm{~K})$ versus IR $(<1 \mathrm{~K})$. The quality of this match is typical for lines of sight with clear views throughout the entire raster. This is good evidence that the emissivity of volcanic lava domes is similar at both IR and MMW wavelengths. (iv) Convolved IR can produce a higher temperature than MMW. Throughout the entire dataset, comparing elevations tends to show some attenuation of the IR due to gas and steam, however occasionally features of the convolved IR read higher in temperature than the MMW radiometry, differing by up to 30 ${ }^{\circ} \mathrm{C}$. This might be due to the changing character of the dome over short time scales $(<30 \mathrm{~s})$ e.g. a minor rockfall revealing heat underneath or localized variations in emissivity due to changing composition of the lava dome, but it is more likely that these features are simply so small that the spatial sampling of the AVTIS raster scan is insufficient to match the convolved IR.

Despite these differences, these data provide strong evidence that MMW radiometric imaging can be used to make brightness temperature measurements of volcanic scenes through obscurants that agree with infrared imaging. However, they also show that brightness temperatures measured with MMW will only give a bulk average and can significantly under-estimate peak temperatures of small thermal features in a volcanic scene. We have not related brightness temperature to physical temperature as may be needed for geophysical modeling. This requires knowledge of the emissivity of lava at $94 \mathrm{GHz}$ and the atmospheric attenuation at the time of data capture which are factors that AVTIS is unable to measure and would have to be determined experimentally in the field and/or laboratory. Another factor is that the absolute calibration of AVTIS radiometric measurements relies on the elevation profile of atmospheric brightness temperature which can be variable. Better absolute calibration may require integration of additional calibration noise sources. Nonetheless the current AVTIS thermal imagery should be attainable in all but the worst weather conditions and provide relative measurements of temperature variations due to volcanic activity when alternative survey techniques fail.

\section{CONCLUSION}

AVTIS is a novel, field portable MMW imaging instrument, specifically designed to record 3D topographic maps and thermal images in virtually all weather conditions. The principal intended application for AVTIS is the monitoring of active volcanic lava domes, but it is also suitable for other applications requiring terrain mapping through obscurants. Data can be collected at long ranges of up to $\sim 6000 \mathrm{~m}$, with only heavy rain inhibiting operation. DEMs are created from GPS referenced radar data which can be collected from multiple viewpoints and subsequently merged.

In this paper we have presented the design and calibration of the instruments radar and radiometric modes. The necessary processing steps required to yield geo-referenced DEMs from radar measurements is explained. As the radar has been developed, data processing has necessarily become more sophisticated and better geared towards optimizing the interpretation of raw AVTIS data. Whilst the basic principles of data processing have remained the same, the development of algorithms has led towards automated retrieval of topographic data. Corrections can now be calculated and applied to the data automatically, a process which previously would take months of manual data manipulation.

The comparison of DEMs enables height and thus volume changes to be quantified, and the errors associated with this are considered, comparing co-located and different viewpoint measurements. Volume change at the Soufriere Hills Volcano, Montserrat was measured and from this an estimated lava extrusion rate was calculated.

In addition to topographic surveying, the calibrated radar data enable the measurement of the normalized radar cross section, $\sigma^{0}$, of the terrain being imaged. We present what we believe to be the first reported measurement of $\sigma^{0}$ at millimeter wavelengths for volcanic terrain. Such information may be valuable for terrain classification and identification of surface features.

Radiometric performance has been validated in the field with comparison to infrared data. Whilst infrared imaging is obscured by cloud, gas and rain, we have shown that MMW thermal imagery is relatively unaffected and yields quantitative temperature measurements.

The validation of AVTIS in field operations on multiple occasions has proven its worth as a practical instrument. Currently we are engaged in the deployment and development of improved versions of the instrument which incorporate fully automatic processing software to enable AVTIS to be used as an operational monitoring tool in a volcano observatory.

\section{ACKNOWLEDGMENT}

The authors would like to thank the large number of people that have contributed in many ways to the development of the AVTIS instrument over the last nine years. We are grateful for help from colleagues at St Andrews, Reading and Lancaster Universities, staff at the Montserrat Volcano Observatory as well as OVSICORI-UNA in Costa Rica for their substantial help in the field. We are also indebted to Patrick Beasley of QinetiQ for technical contributions during hardware development.

\section{REFERENCES}

[1] Sparks et al., "Magma production and growth of the lava dome of Soufriere Hills Volcano, Montserrat, West Indies, November 1995 to December 1998", Geophys.Res. Lett., vol. 25, pp. 3421-3424, 1998.

[2] Jones, L., "Monitoring landslides in hazardous terrain using terrestrial LiDAR: an example from Montserrat", Q.J. Eng. Geol. Hydrogeol., vol. 39, pp. 371-373, 2006.

[3] Harris A., Dehn, J., Patrick, M., Calvari. S., Ripepe, M., Lodato, L., "Lava effusion rates from hand-held thermal infrared imagery: an example from the June 2003 effusive activity at Stromboli", Bull. Volcanol., vol. 68, pp. 107-117, 2005.

[4] Watts, R.B. Herd, R.A., Sparks, R.S.J. and Young, S.R. "Growth patterns and emplacement of the andesitic lava dome at Soufriere Hills Volcano, Montserrat", The eruption of Soufriere Hills Volcano, Montserrat from 1995 to 1999. Geol. Soc. London, Memoir 21, pp.115$152,2002$. 
[5] Lu, Z., Fielding, E., Patrick, M.R. and Trautwein, C.M., "Estimating lava volume by precision combination of multiple baseline spaceborne and airborne interferometric synthetic aperture radar: the 1997 eruption of Okmok volcano, Alaska", IEEE Trans. Geosci. Remote Sens., vol. 41, no.6, pp.1428-1436, June 2003, doi:10.1109/TGRS.2003.811553

[6] Wadge, G. et al, "ERS SAR interferometry of an erupting volcano on a tropical island: Soufrière Hills Volcano, Montserrat." in Proc. IEEE IGARSS, 1999, pp. 2170-2172.

[7] Marzano, F.S., Barbieri, S., Picciotti, E. and Karlsdottir, S., "Monitoring Subglacial Volcanic Eruption Using Ground-Based C-Band Radar Imagery", IEEE Trans. Geosci. Remote Sens., vol. 48, no.1, pp.403-414, Jan. 2010, doi:10.1109/TGRS.2009.2024933

[8] Voge, M and Hort, M.,"Installation of a Doppler Radar Monitoring System at Merapi Volcano, Indonesia", IEEE Trans. Geosci. Remote Sens., vol. 45, no.1, pp.251-271, Jan.2009, doi:10.1109/TGRS.2008.2002693

[9] Casagli, N., Tibaldi, A., Marri,, A., Del Ventisette, C., Apuani, T., Guerri, L., Fortuny-Guasch, J., Tarqui, D., "Deformation of Stromboli Volcano (Italy) during the 2007 eruption revealed by radar interferometry, numerical modelling and structural field data", $J$. Volcanol. Geotherm. Res., vol. 182, pp.182-200, 2009.

[10] Nico, G., Leva, D., Fortuny-Guasch, J., Antonello, G. and Tarchi, D., "Generation of digital terrain models with a ground-based SAR system", IEEE Trans. Geosci. Remote Sens., vol. 43, no.1, pp.45-49, Jan. 2005, doi: 10.1109/TGRS.2004.838354

[11] Wadge, G., Macfarlane, D.G., Robertson, D.A., Hale, A.J., Pinkerton, H., Burrell, R.V., Norton, G.E. and James, M.R., "AVTIS: a novel millimetre-wave ground based instrument for volcano remote sensing", J. Volcanol. Geotherm. Res., vol. 146, pp.307-318, 2005.

[12] Macfarlane, D.G., Wadge, G., Robertson, D.A., James, M.R., Pinkerton, H., "Use of a portable topographic mapping millimetre wave radar at an active lava flow", Geophys. Res. Lett., vol. 33, L03301, 2006. doi:10.1029/2005GL025005.

[13] Wadge, G., Macfarlane, D.G., James, M.R., Odbert, H.M., Applegarth. L.J., Pinkerton, H., Robertson, D.A., Loughlin, S.C., Strutt, M., Ryan, G., Dunkley, P.N. (2006) Imaging a growing lava dome with a portable radar. EOS, AGU 87/23, 226-228

[14] Wadge, G., Macfarlane, D.G., Odbert, H.M., James, M.R., Hole J.K., Ryan, G., Bass, V., De Angelis, S., Pinkerton, H., Robertson, D.A. and Loughlin, S.C. (2008). Lava dome growth and mass wasting measured by a time series of ground-based radar and seismicity observations. $J$. Geophys. Res. 113, B08210, doi:10.1029/2007JB005466.

[15] Robertson, D.A. \& Macfarlane, D.G., "A 94GHz dual-mode imaging "radarometer" for remote sensing", Passive Millimeter Wave Imaging Technology IX, Proc. SPIE 6211, Orlando, USA, 17-21 April, 2006, doi: 10.1117/12.669668.

[16] Macfarlane, D.G. \& Robertson, D.A., "A $94 \mathrm{GHz}$ dual mode imager combining FMCW radar and passive radiometry for remote sensing", Proc. Intl. Radar Symposium, Berlin, Germany, pp. 153-157, 2005.

[17] M. A. Richards, Fundamentals of Radar Signal Processing. McGrawHill, New York, 2005.

[18] Rosker, M.J. and Wallace, H.B, (2007), "Imaging Through the Atmosphere at Terahertz Frequencies", IEEE/MTT-S International Microwave Symposium, pp 773 - 776, 2007, doi: 10.1109/MWSYM.2007.380054

[19] N. Currie, R. Hayes, and R. Trebits, Millimeter Wave Radar Clutter, Artech House, 1992, Ch 3.

[20] F. T. Ulaby, A. El-Rouby, E. S. Li, R. D. De Roo, R. J. Wellman, and H. B. Wallace, "95-GHz Scattering by Terrain at Near-Grazing Incidence", IEEE Trans. on Antennas and Propagation, vol.46, no. 1, pp. 3-12, 1998.

[21] Ulaby, F.T.,Moore, R.K. \& Fung, A.K., Microwave Remote Sensing: Active and Passive (Volume I), Artech House, 1981, Ch 6.

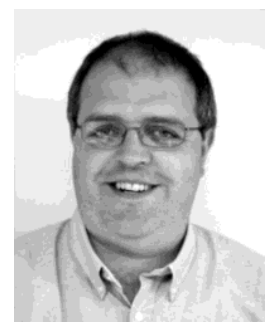

David G. Macfarlane (M'04) received a BSc from the University of Glasgow, Glasgow, in 1995 and an MSci (Hons) in Theoretical Physics followed by a $\mathrm{PhD}$ in millimeter wave imaging from the University of St Andrews, St Andrews, Fife in 1999 and 2002 respectively. Since 2002 he has been a Research Fellow with the Millimetre Wave and EPR Group, University of St Andrews. He is currently managing an EPSRC funded outreach programme on the applications of millimeter wave technology as well as continuing to work on the development of the AVTIS instruments. His research interests include millimeter wave radar, radiometry, imaging and remote sensing of terrain.

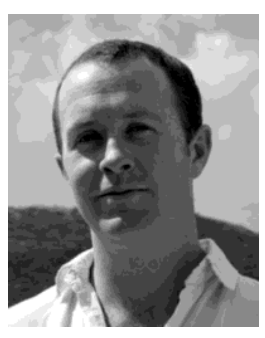

Henry Odbert received the MEarthSci degree from Oxford University in 2005 and a Ph.D. degree from The University of Reading in 2009. He works at the Montserrat Volcano Observatory as the Ground Deformation Volcanologist and Deputy Director and is a Research Fellow at The University of the West Indies Seismic Research Centre, Trinidad. His main research interests concern eruptive processes of volcanoes, particularly the development of tools and analyses for monitoring. He has worked with the AVTIS team since 2005 .

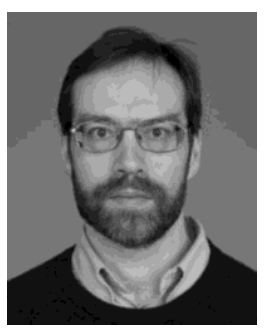

Duncan A. Robertson (S'91-M'094) was born in Aberfeldy, UK, in 1969. He received a BSc(Hons) in Physics and Electronic and a $\mathrm{PhD}$ in millimetre wave physics from the University of St Andrews, St Andrews, Fife, UK, in 1991 and 1994 respectively.

From 1994 to 1999 he was a Research Fellow with the Millimetre Wave Group, University of St Andrews, under contract to DERA Malvern, working on battlefield millimetre wave systems. Between 1999 and 2000 he was a Principal Microwave Engineer with Racal-MESL, Edinburgh, UK, working on high power radar duplexers. From 2000 to 2004 he worked in the Photonics Innovation Centre, University of St Andrews, commercializing millimetre wave technology. Since 2004 he has been a Research Fellow with the Millimetre Wave and EPR Group, University of St Andrews. His research interests include millimetre wave radar, radiometry, imaging, electron paramagnetic resonance instrumentation, materials characterisation (ferrites, dielectrics and absorbers) and antennas (corrugated horns, quasi-optics and non-mechanical beam steering). Dr Robertson is a Chartered Physicist, a member of the Institute of Physics, a member of the Institute of Engineering and Technology and a member of SPIE.

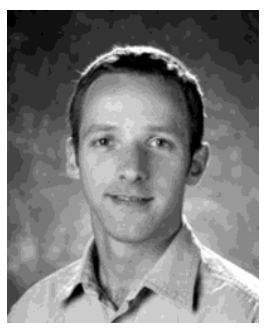

Mike James does not have a bio, blah, blah, blah, , blah, blah, blah, , blah, blah, blah, , blah, blah, blah, , blah, blah, blah, , blah, blah, blah, , blah, blah, blah, , blah, blah, blah, , blah, blah, blah, , blah, blah, blah, , blah, blah, blah, , blah, blah, blah, , blah, blah, blah, , blah, blah, blah, , blah, blah, blah, , blah, blah, blah, , blah, blah, blah, , blah, blah, blah, , blah, blah, blah, , blah, blah, blah, , blah, blah, blah, , blah, blah, blah, , blah, blah, blah, , blah, blah, blah, , blah, blah, blah, , blah, blah, blah, , blah, blah, blah, , blah, blah, blah, , blah, blah, , blah, blah, blah, , blah, blah, blah, , blah, blah, blah, , blah, blah, blah, , blah, blah, blah, , blah, blah, blah, , blah, blah, blah, , blah, blah, blah, ,

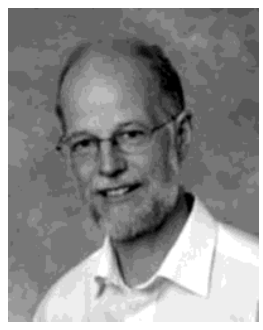

Harry Pinkerton does not have a bio either, blah, blah, blah, , blah, blah, blah, , blah, blah, blah, , blah, blah, blah, , blah, blah, blah, , blah, blah, blah, , blah, blah, blah, , blah, blah, blah, , blah, blah, blah, , blah, blah, blah, , blah, blah, blah, , , blah, blah, blah, blah, blah, blah, , blah, blah, blah, , blah, blah, blah, , blah, blah, blah, , blah, blah, blah, , blah, blah, blah,, blah, blah, blah, , , blah, blah, blah, , blah, blah, blah, , blah, blah, blah, , blah, blah, blah, , blah, blah, blah, , blah, blah, blah, , blah, blah, blah, , blah, blah, blah, ,

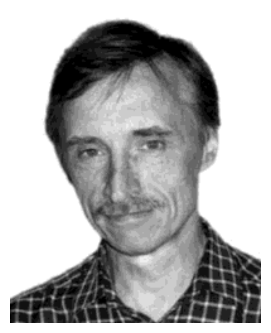

Geoff Wadge received his $\mathrm{BSc}$ and $\mathrm{PhD}$ degrees at Imperial College, London in 1971 and 1974. He works at the National Centre for Earth Observation at the University of Reading, U.K. as a professorial research fellow, and is currently Director of the Environmental Systems Science Centre there. His main research interest is in volcanology, particularly the remote sensing of volcanic processes using radar. He has been the P.I. for the AVTIS project and is Chairman of the Scientific Advisory Committee for the Soufriere Hills Volcano on Montserrat. 